Supporting Information

\title{
An Interactive Interface for Graph-Based Analyses of Dynamic H-bond Networks: Application to Spike Protein S
}

\author{
Malte Siemers ${ }^{1}$ and Ana-Nicoleta Bondar ${ }^{1 *}$ \\ ${ }^{1}$ Freie Universität Berlin, Department of Physics, \\ Theoretical Molecular Biophysics, \\ Arnimallee 14, D-14195 Berlin, Germany
}

*Correspondence to nbondar@zedat.fu-berlin.de 
Supporting Information contains Figures S1-S28 and References.

The files for installing the interactive Bridge2 interface are provided as a separate Supporting Information file. 


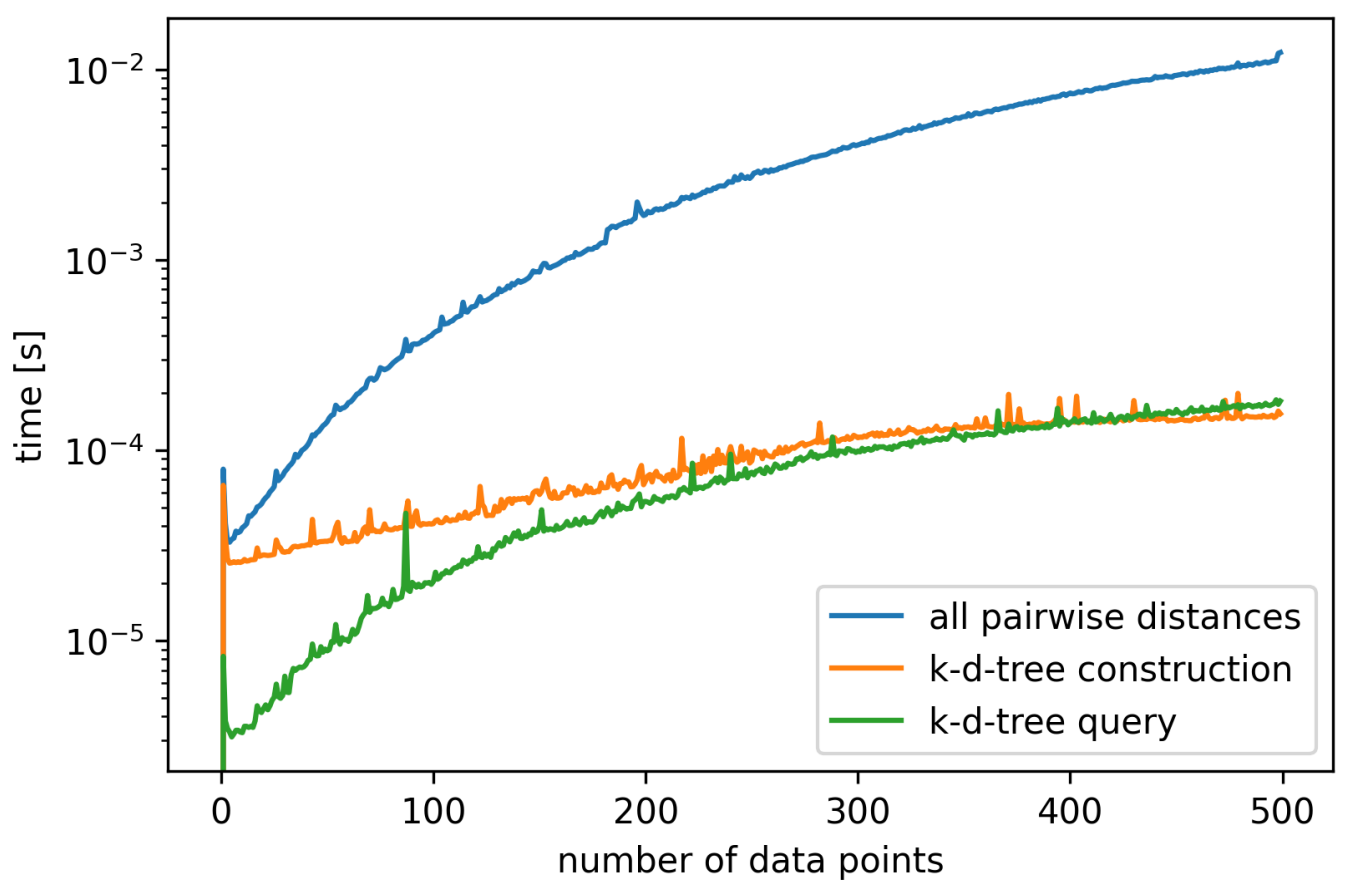

Figure S1. Test of the computational efficiency of the k-d-tree approach used to identify $\mathrm{H}$ bond neighbors. We used 499 independent data sets, each containing from 2 to 500 points generated randomly within the unit cube. We used the Python packages numpy and time to calculate the time required to construct the k-d-tree for the respective data set (orange profile), and to query the resulting tree to extract all neighbors within a distance of 0.03 (green profile). Bridge uses the cKDTree class of the Python scipy package to construct and query k-d-trees. The profile computed using the naïve implementation shows the time needed to compute every pairwise distance using the distance matrix implementation of the numpy package (blue profile). 


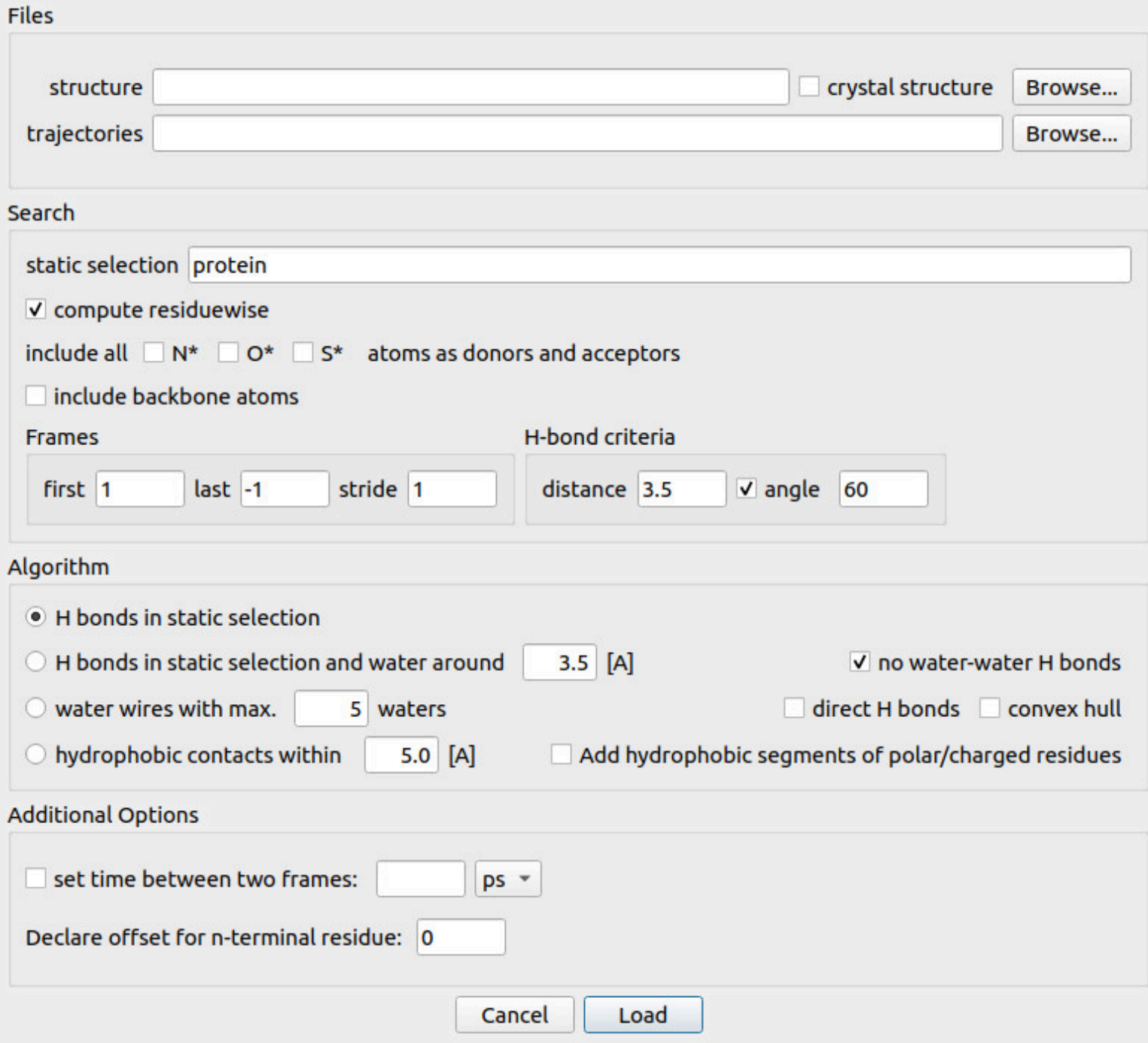

Figure S2. The New Analysis window of Bridge2. Under 'Structure', Bridge2 expects a standard Protein Data Bank coordinate file, or a standard Protein Structure File. Trajectories can be read under 'trajectories' in the order required for analyses. Selecting 'crystal structure' instructs Bridge 2 that there are no $\mathrm{H}$ atoms, and $\mathrm{H}$-bonds will be computed with a distance criterion. The typical static selection for analyses is 'protein'. We recommend interactions to be computed residuewise, as an atomwise computation may result in very large numbers of nodes. All settings for $\mathrm{H}$-bond criteria, reading step for the trajectory, and inclusion of waters in computations, can be changed by the user. The computation is initiated by pressing the button 'Load'.

Additional options for the initiation of the graph computation include a declaration of the offset of the N-terminal amino acid residue of the protein, and of the time between two consecutive frames of the simulation trajectory. 
Acceptor atoms

ND1, NE2, OD1, OD2, OE1, OE2, OG, $\mathrm{OG} 1, \mathrm{OH}, \mathrm{OH} 2, \mathrm{OW}, \mathrm{SD}, \mathrm{SG}$

Water oxygen atoms

(resname TIP3 and name $\mathrm{OH} 2$ ) or (resname $\mathrm{HOH}$ and name O)

\section{Cancel}

Reset

Save

\section{Donor atoms} $\mathrm{NH} 2, \mathrm{NZ}, \mathrm{OD} 1, \mathrm{OD} 2, \mathrm{OE} 1, \mathrm{OE} 2, \mathrm{OG}$,

$\mathrm{OG} 1, \mathrm{OH}, \mathrm{OH} 2, \mathrm{OW}, \mathrm{SG}$

N16, ND1, ND2, NE, NE1, NE2, NH1,

Figure S3. Default atom types of MDAnalysis ${ }^{1}$ which are used by the Bridge2 interface to compute $\mathrm{H}$-bonds. Additional atom types would need to be declared in this window, before the New Analysis dialog is used. 


\section{令士 $Q$ 田}

\begin{tabular}{l} 
Layout \\
Projection plane \\
best view PCA plane ZY plane XY plane \\
frame \\
Colors \\
per segment PROA seagreen \\
single color seagreen \\
centrality measures \\
normalized $\checkmark$ averaged across frames \\
betweenness centrality \\
degree centrality \\
Sizes unique shortest paths for single coordinate set \\
nodes: \\
edges: \\
edge labels: \\
Labels \\
nodes: $\checkmark$ residue names \\
edges: \\
\hline
\end{tabular}

Figure S4. The Menu, and the Layout window of the interactive Bridge2 interface.

The upper image shows the four buttons used to, respectively, read the coordinate files to be analyzed, and thus initiate a new analysis; open an existing Bridge 2 analysis file; save the results of the Bridge 2 analysis; save the results of the Bridge 2 analysis under a different name. The Menu will remain available throughout the entire Bridge2 computation.

The second image shows the Layout window, which will remain open during computation with criteria chosen by the user (see the New Analysis window illustrated in Figure S2 below). On the left bottom corner, the Layout window will report interactively the current status of the computation.

By default, the graph will be shown in the best view PCA plane. The user can select the ZY or XY planes.

By default, graph nodes are covered according to the segment identification to which amino acid residues belong. These colors can be chosen from the Colors menu. Alternatively, the 
color of the nodes can be changed to represent centrality values enabled in the Layout window.

The user can modify the font size of the node identification, the thickness of the graph edges, and the font size for the numbers that indicate values for the edges, such as the occupancy or the number of waters in the bridge.

The options under Labels are used to show on the graph information of interest to the user. When active, the default option 'residue names' gives the name and ID of each amino acid residue part of the graph. As this can lead to very crowded graphs, the user may choose to switch off the labels for the nodes. Edges can be chosen to indicate occupancy values, endurance times for water wires, or the average number of water molecules in the wire. 


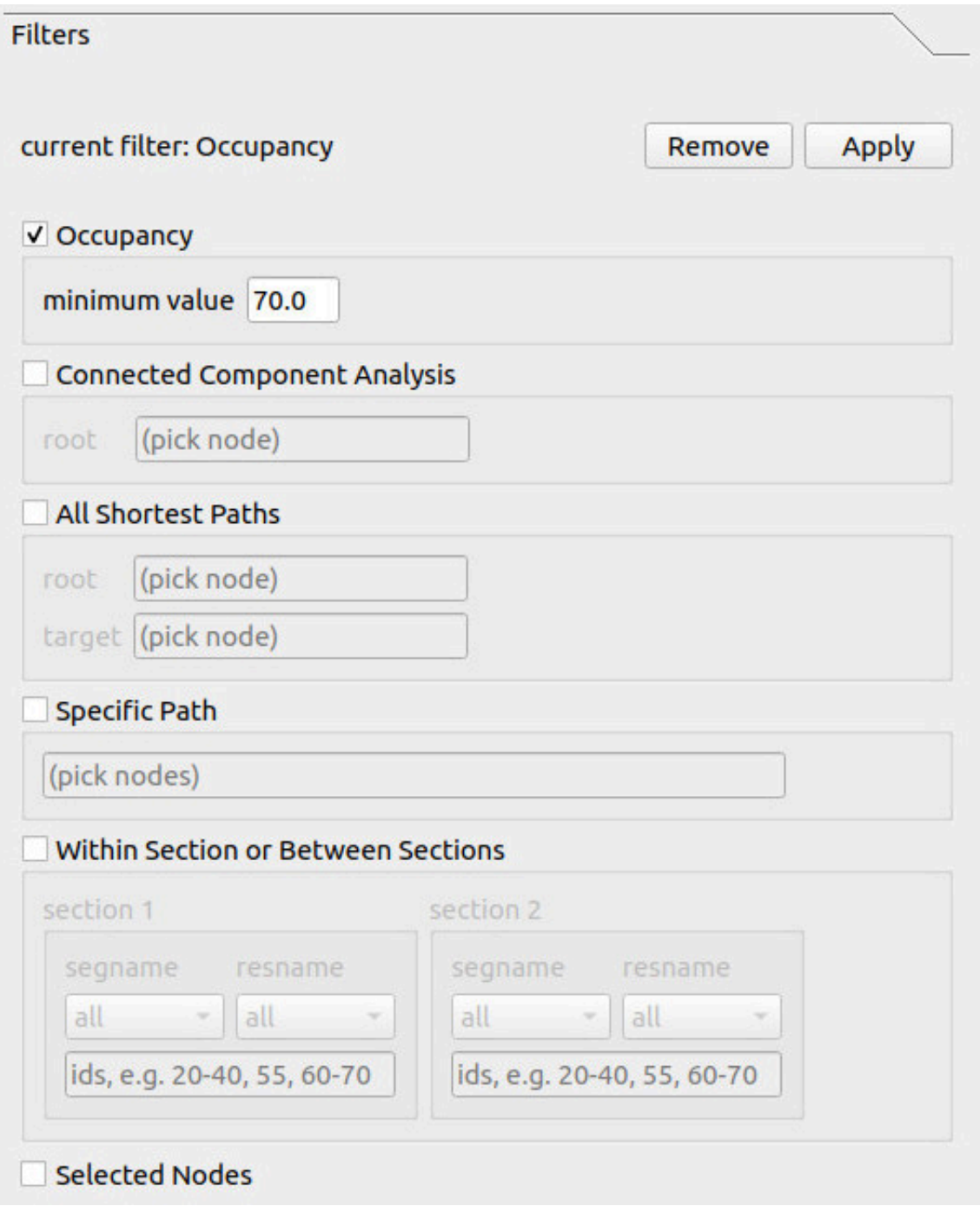

Figure S5. The Filters window of Bridge2. Filters can be applied or removed, and the current status filter is displayed under 'current filter'. For a filter to be applied or removed, the appropriate button needs to be selected.

The Occupancy Filter expects a value $\leq 100$, with a value of 100 corresponding to $\mathrm{H}$-bonds present in all coordinate sets included in the computation.

The Connected Component Analysis is performed for a root node that can be selected with a mouse click on the graph node of interest, or with the node identification provided in the corresponding box.

Graph nodes are identified as SegmentID-ResidueName-ResidueNumber, e.g., PROAGLU-1.

The Specific Path Filter requires all nodes of the path to be specified in the correct order.

The Within Section or Between Sections Filter requires a selection of subsets of nodes of the graph, chosen with the corresponding selections available in this Filter.

The Selected Nodes Filter reports the $\mathrm{H}$-bonds or $\mathrm{H}$-bond networks that involve a previously selected set of nodes, such as, for example, from a previous computation of motifs. 


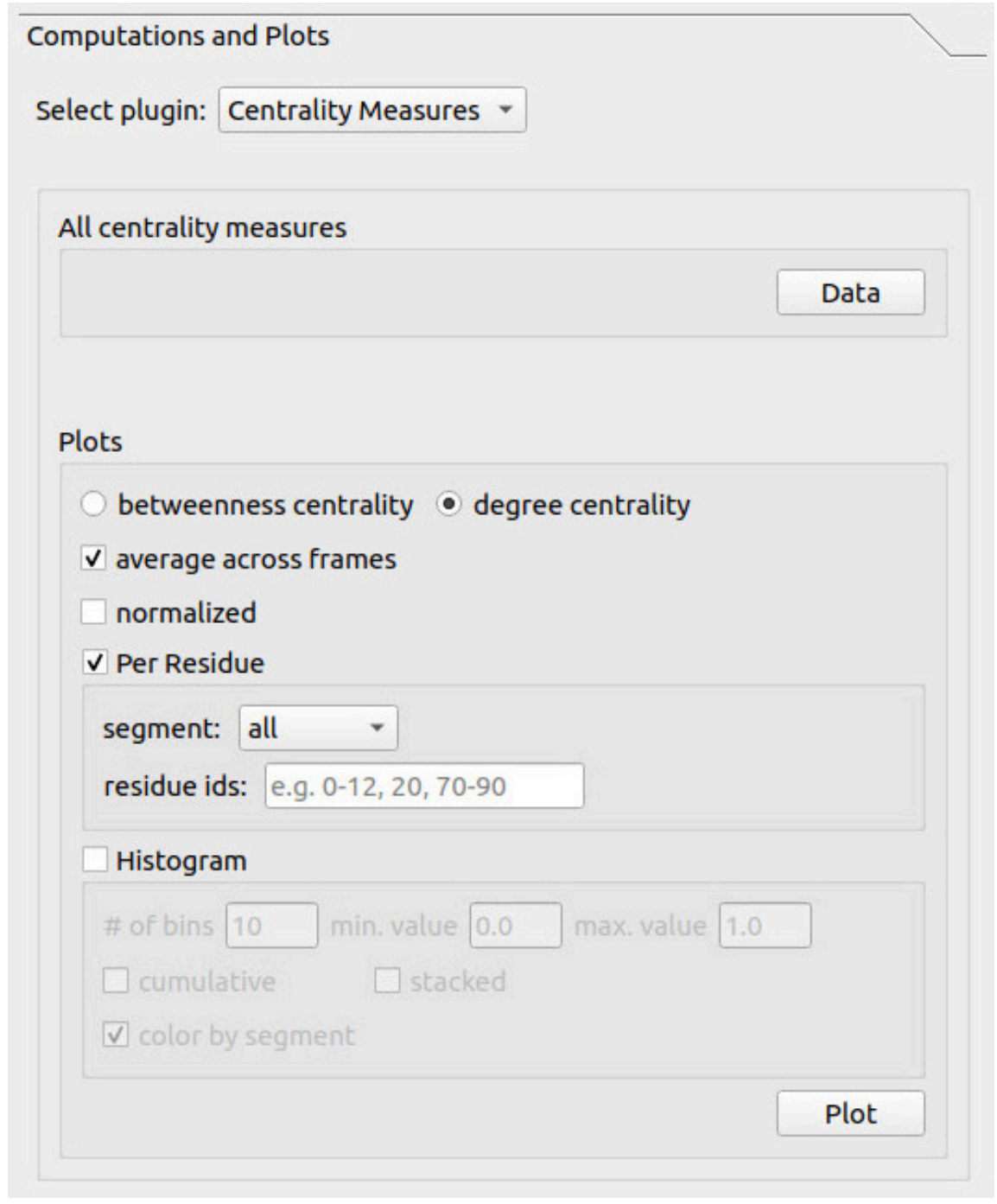

Figure S6. The Centrality Plugin of the Computations and Plots Window. Here, the user can perform, plot, and export $\mathrm{BC}$ and $\mathrm{DC}$ values for a graph of $\mathrm{H}$-bonds or hydrophobic interactions computed previously.

'All centrality measures' reports under Data an ascii file that contains the summary of the results for all $B C$ and $D C$ computations enabled in the Bridge 2 interface. The ascii file can be saved as an external text file for further analyses and plotting.

'Plots' enables computations of selected BC and DC computations as of interest to the user. Results of these computations may be represented as a histogram. All data may be plotted using the plotting tool of Bridge2, and plots may be saved as external files. 

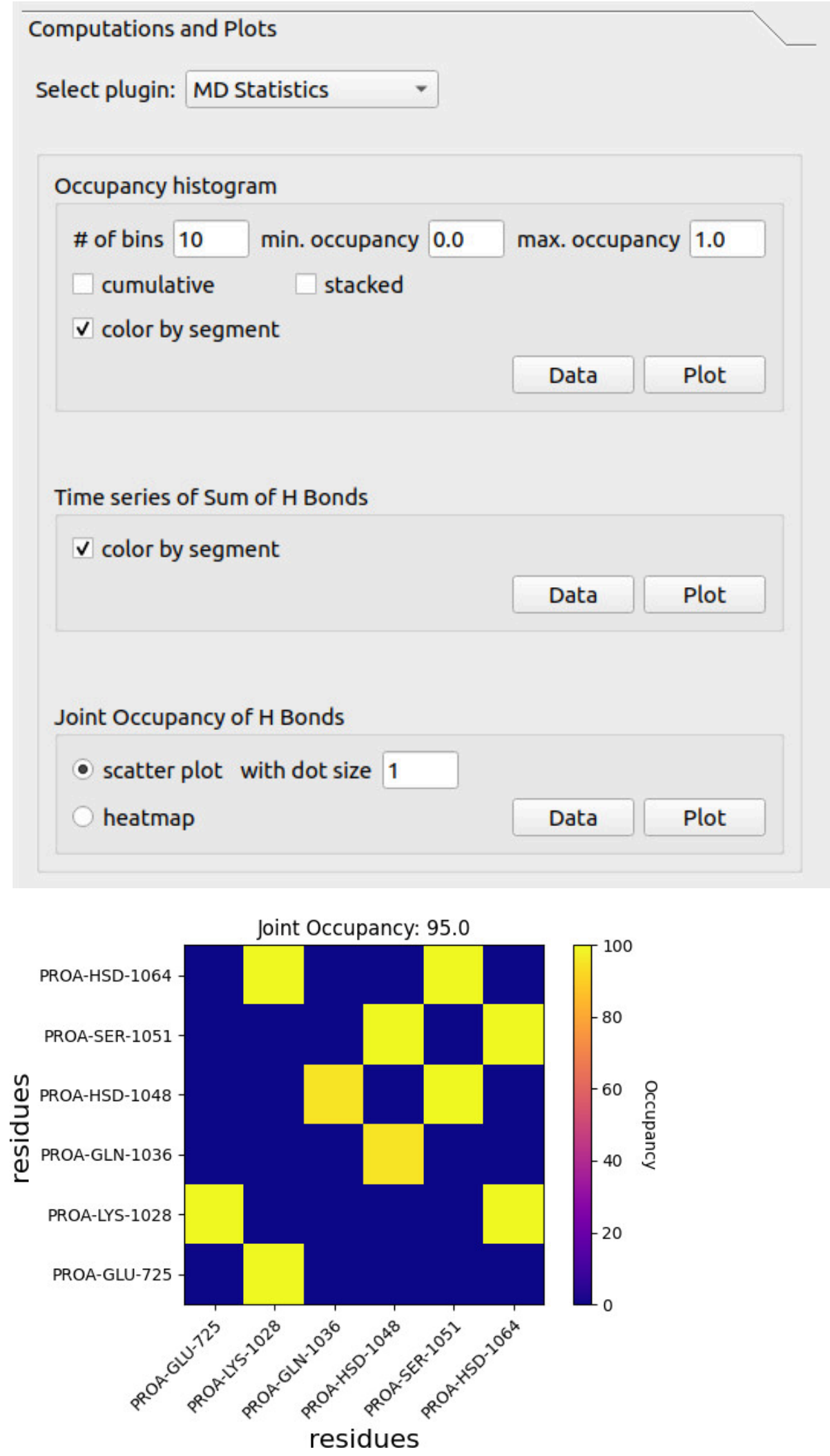

Figure S7. The MD Statistics Plugin of the Computations and Plots Window. This plugin, displayed in the image on the top of the Figure, enables computations of histograms and time series of interactions included in the graphs. Data obtained with the MD Statistics Plugin can be plotted interactively, or it can be exported as external ascii file.

'Occupancy histogram' computes cumulative or stacked integrals using the bins and interval values declared by the user. 'Color by segment' enables bars of the histogram to be colored 
according to the segment IDs read with the structure, such as different segment names for a protein composed of several chains.

'Number of connections time series' provides a time series of the number of connections for the active selection. If the time unit between two consecutive coordinate sets was provided upon initiating the computation, the time series will display the time computed accordingly. Otherwise, Bridge2 will report the number of coordinate sets (frame) on the horizontal axis of the plot.

Given a previously selected H-bond paths, 'Joint Occupancy of H-bonds' reports the time series of the joint occupancy value as true (1) when all $\mathrm{H}$-bond segments of the path are present simultaneously, and false (0) otherwise.

In a heatmap representation of the $\mathrm{JO}$ values of an $\mathrm{H}$-bond path, amino acid residues part of the path are ordered according to their residue number, and the occupancy value given for each segment of the path -i.e., for each pair of the path, with the JO value given title of the heatmap. The image on the bottom of thus figure shows an example of a heat map representation for an $\mathrm{H}$-bonded path, computed from 20 coordinate snapshots of the simulations reported here. 


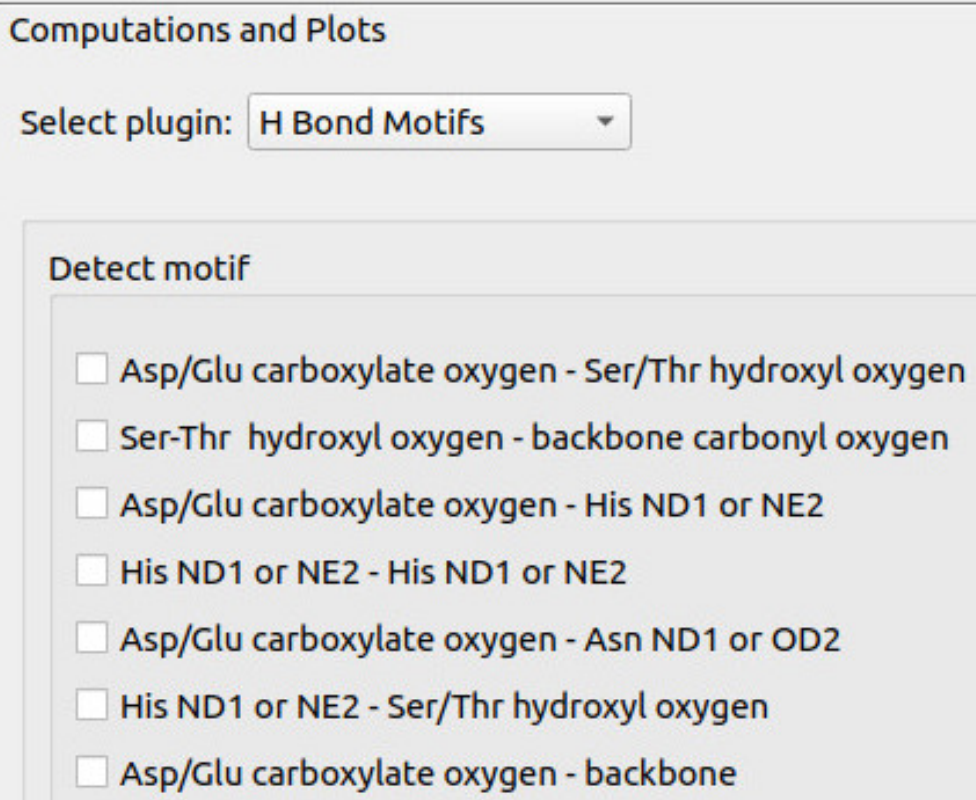

Figure S8. The H-bond Motifs plugin of the Computations and Plots Window. The user can choose between one of the seven types of motifs, or any combination of these motifs. To be active, the Motifs Plugin requires a previously computed $\mathrm{H}$-bond graph with an atomwise detection of the H-bonds.

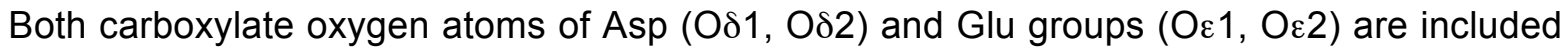
in computations.

For Ser and Thr groups, we include atoms $O \gamma$ and $O \gamma 1$, respectively.

Histidine amino acid residue names included in computations are HSE, HSD, HSP, and $\mathrm{HIS}$; regardless of the histidine amino acid residue name, both the $\mathrm{N} \delta 1$ and $\mathrm{N} \varepsilon 2$ atoms are included in computations of $\mathrm{H}$-bonds.

Backbone atoms included in the computation are oxygen atoms of the backbone carbonyl groups, and nitrogen atoms of the amide groups. 
Bridge2 analysis summary from 24/01/2021 20:27:29

-- ANALYSIS PARAMETER --

- Files -

structure: example.psf

trajectories: example.dcd

crystal structure: No

- Search -

static selection: protein

residuewise: No

included donor atom names: OH, OW, OE2, NH2, N16, NH1, OG1, ND1, NE, OD2, NE1, OH2, OD1, SG, ND2, NE2, OG, NZ, OE1

included acceptor atom names: OH2, OH, OD1, OW, OE2, SG, OG1, OD2, ND1, NE2, SD, OG, OE1

included all oxygen: No

included all nitrogen: No

included all sulphur: No

backbone atoms: No

disulphide bridges: No disulphide bridges included in the search

frames: from 0 to -1 with step 1 , resulting in 20 frames

$\mathrm{H}$ bond distance: 3.5

$\mathrm{H}$ bond angle: 60.0

- Algorithm -

$\mathrm{H}$ bonds in selection

- Additional Options -

time between frames: No time specified

starting $n$-terminal residue: 0

-- FILTER --

No filters applied.

--- RESULTS --

partner1:partner2 occupancy endurance_time

PROA-TYR-37-OH:PROA-LYS-195-NZ 100.0

PROA-GLN-52-OE1:PROA-THR-274-OG1 $\quad 95.0 \quad 17$

PROA-GLU-96-OE1:PROA-ASN-99-ND2 2500

$\begin{array}{lll}\text { PROA-GLU-96-OE1:PROA-ASN-99-ND2 } & 90.0 & 9 \\ \text { PROA-GLU-96-OE2:PROA-ARG-190-NH2 } & 100.0 & 20\end{array}$

$\begin{array}{lll}\text { PROA-GLU-96-OE2:PROA-ARG-190-NH2 } & 100.0 & 20 \\ \text { PROA-ASN-99-OD1:PROA-ARG-102-NH1 } & 100.0 & 20\end{array}$

PROA-ASP-111-OD2:PROA-LYS-113-NZ $100.0 \quad 20$

PROA-LYS-129-NZ:PROA-GLU-169-OE2 $100.0 \quad 20$

PROA-ARG-34-NH1:PROA-GLU-191-OE2 $\quad 95.0 \quad 17$

PROA-TYR-91-OH:PROA-GLU-191-OE2 $\quad 100.0 \quad 20$

PROA-LYS-206-NZ:PROA-GLU-224-OE1 $\quad 95.0 \quad 18$

PROA-LYS-202-NZ:PROA-ASP-228-OD1 $75.0 \quad 5$

Figure S9. Illustration of an ascii file that reports the summary of $\mathrm{H}$-bond analyses for a test computation with 20 coordinate sets from the simulation of spike protein $\mathrm{S}$, with up to five water molecules in $\mathrm{H}$-bonded wires between protein sidechains, and with standard distance and angle $\mathrm{H}$-bond criteria. 

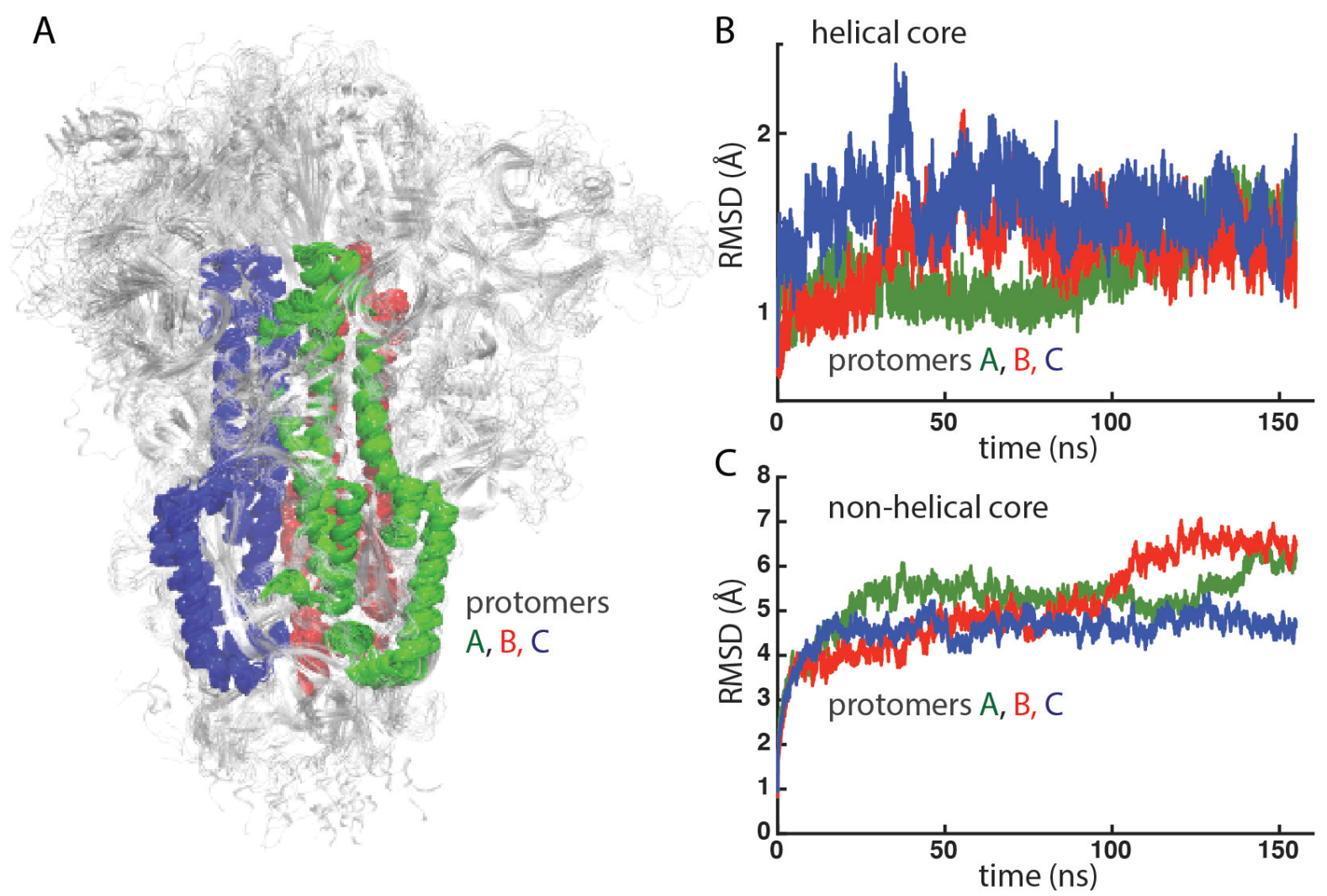

Figure S10. C $\alpha$ RMSD profiles computed for the three protomers of protein $S$ indicate structural stability of the helical core of the protein. (A) Molecular graphics of the spike protein indicating the protein segments included in the selection denoted here as core helical segments. The core helical segments comprise, for each of the protomers, amino acid residues D737 to G757, R765 to A783, S816 to V826, T866 to G891, P897 to S968, and V976 to L1034. Protomer A, B, and C helical segments are colored green, red, and blue, respectively. Non-core helical segments are the remaining segments of each protomer; for clarity of the image, these segments are shown as thin, transparent, white cartoons. (B) C $\alpha$ RMSD profiles computed for the core helical segments of protein $S$. (C) $\mathrm{C} \alpha$ RMSD profiles for the non-core helical segments of protein $\mathrm{S}$. All molecular graphics were prepared with VMD. ${ }^{2}$ 


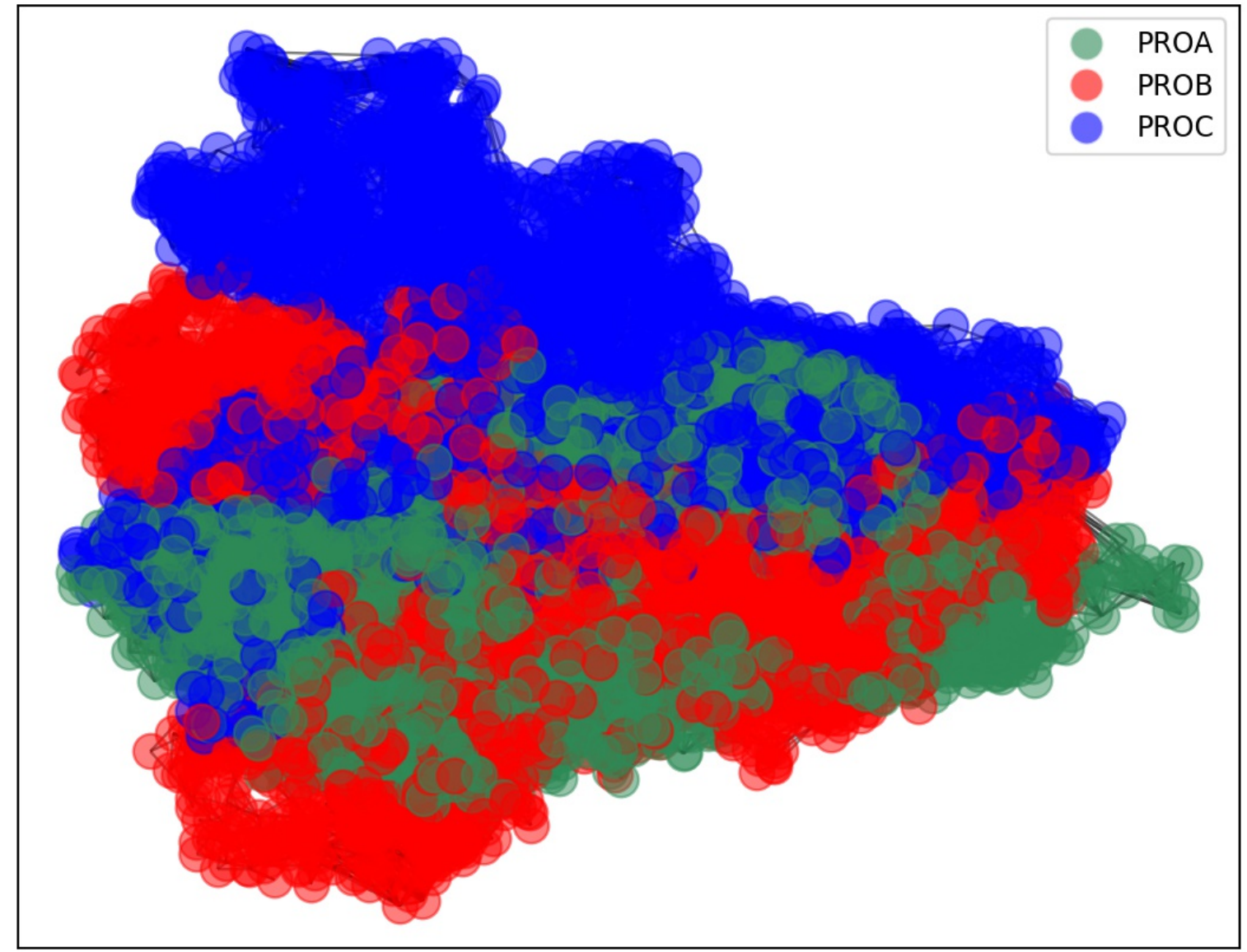

Figure S11. The interactive Bridge2 display of an atom-wise computation of the sidechainsidechain and sidechain-backbone $\mathrm{H}$-bonds of protein $\mathrm{S}$, without any occupancy filter. Bridge 2 assigns default colors to the three protein segments as declared in the protein structure file used to read the MD trajectory, PROA, PROB and PROC, corresponding to protomers $A, B$ and $C$ of the spike protein $S$. The graph is overly crowded due to the large number of $\mathrm{H}$-bonds identified. 


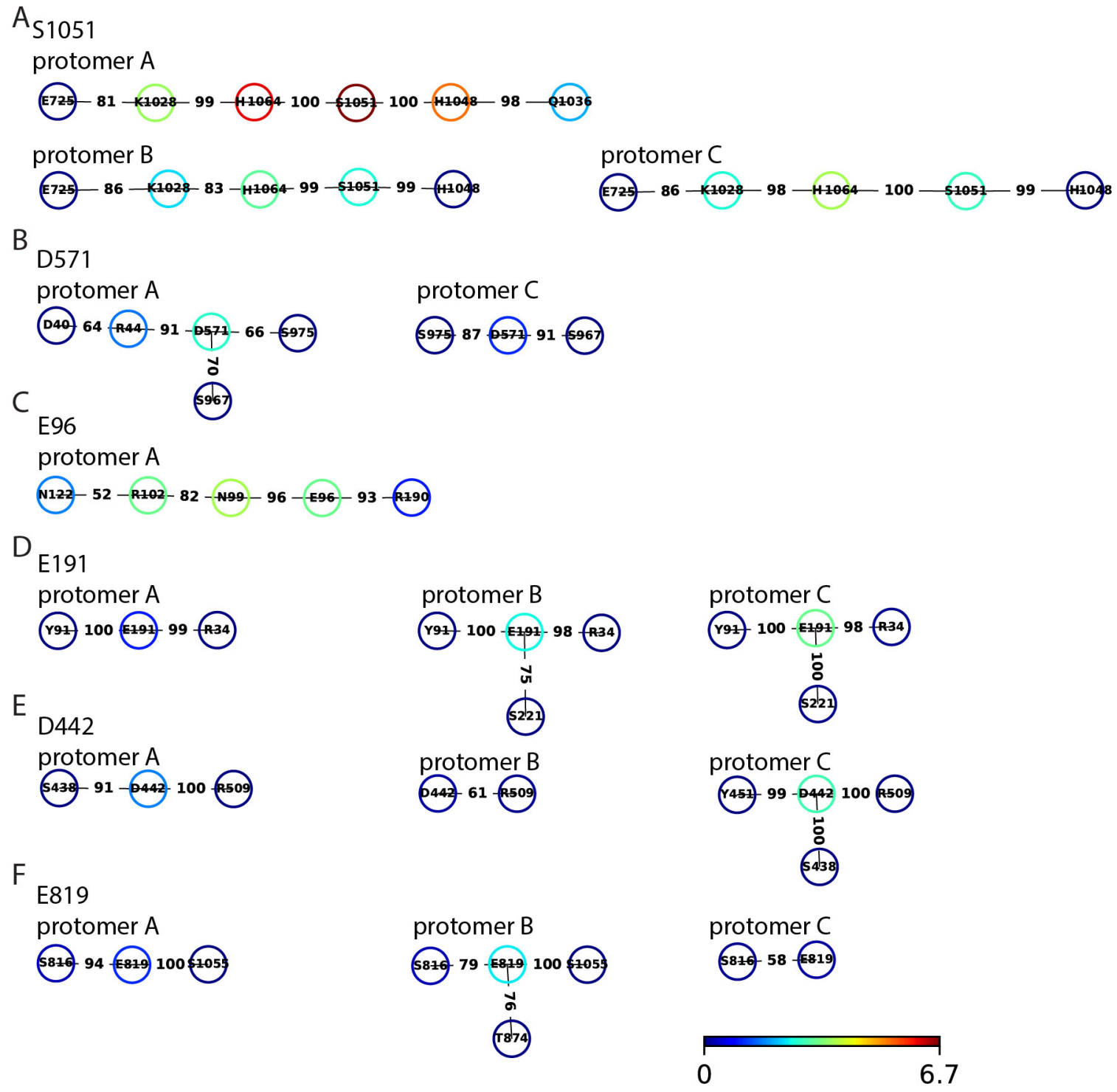

Figure S12. Illustration of the local $\mathrm{H}$-bond networks identified, in the $\mathrm{H}$-bond graph filtered at $50 \%$, for groups that have high occupancy. Colors of the nodes, and the color bar, indicate $\mathrm{BC}$ values computed for the complete $\mathrm{H}$-bond graph for $\mathrm{H}$-bonds between sidechains, and thus $\mathrm{H}$-bonds are not distinguished as intra- vs. inter-protomer. The protomer is indicated for the group used as root node in the search for local $\mathrm{H}$-bond clusters. (A-B) The local H-bond networks of S1051 (panel A) and D571 (panel B). D571 of protomer $B$ is not part of the graph filtered at $50 \%$. (C) Local $\mathrm{H}$-bond network of E96 in protomer $A$; the $50 \%$ occupancy graph lacks $\mathrm{H}$-bonds of protomers $\mathrm{B}$ and $\mathrm{C}$ E96. (D-F) Local H-bond networks of E191 (panel D), D442 (panel E), and E819 (panel F). In panel F, note that the $\mathrm{H}$-bond between $\mathrm{S} 816$ and $\mathrm{E} 819$ is present in all protomers. 


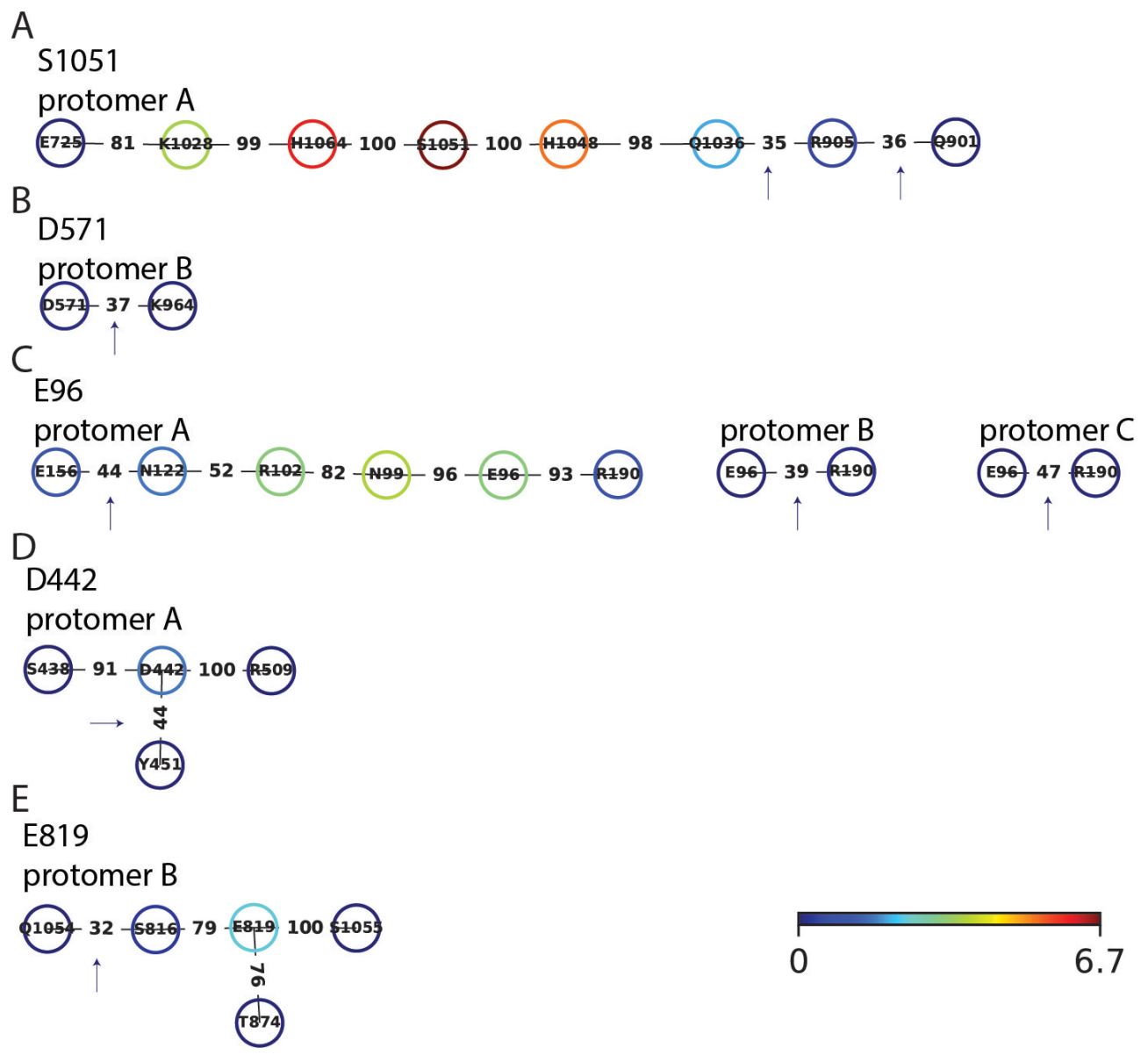

Figure S13. Illustration of local $\mathrm{H}$-bond clusters that become more extended when lowering the $\mathrm{H}$-bond occupancy threshold to $30 \%$. Arrows indicate $\mathrm{H}$-bonds that are present in the $30 \%$ occupancy graph, and not in the corresponding $50 \%$ occupancy graphs presented in Figure S12. (A) The S1051 cluster in protomer A. The S1051 clusters of protomer B and C, remained the same as those presented in Figure S12A. (B) The D571 cluster in protomer B; in protomers $A$ and $C$, the D571 cluster remained the same as in Figure S12. (C) The E96 $\mathrm{H}$-bond cluster recruits $\mathrm{E} 156$ in protomer $\mathrm{A}$, whereas in protomers $\mathrm{B}$ and $\mathrm{C}$ the $\mathrm{H}$-bond between E96 and R190 is sampled. (D) The local H-bond cluster of D442; in protomers B and $\mathrm{C}$, the $\mathrm{H}$-bonding of D442 is the same as in to $50 \%$ occupancy graphs presented in Figure S12. (E) At 30\% $\mathrm{H}$-bond occupancy in protomer $\mathrm{B}$, the local $\mathrm{H}$-bond graph of $\mathrm{E} 819$ extends to include Q1054; in protomers A and C, the H-bond graph of E819 remains the same as in the $50 \%$ occupancy graphs In all three protomers, the local cluster of E191 is the same in the $30 \%$ vs. $50 \% \mathrm{H}$-bond occupancy graphs. 


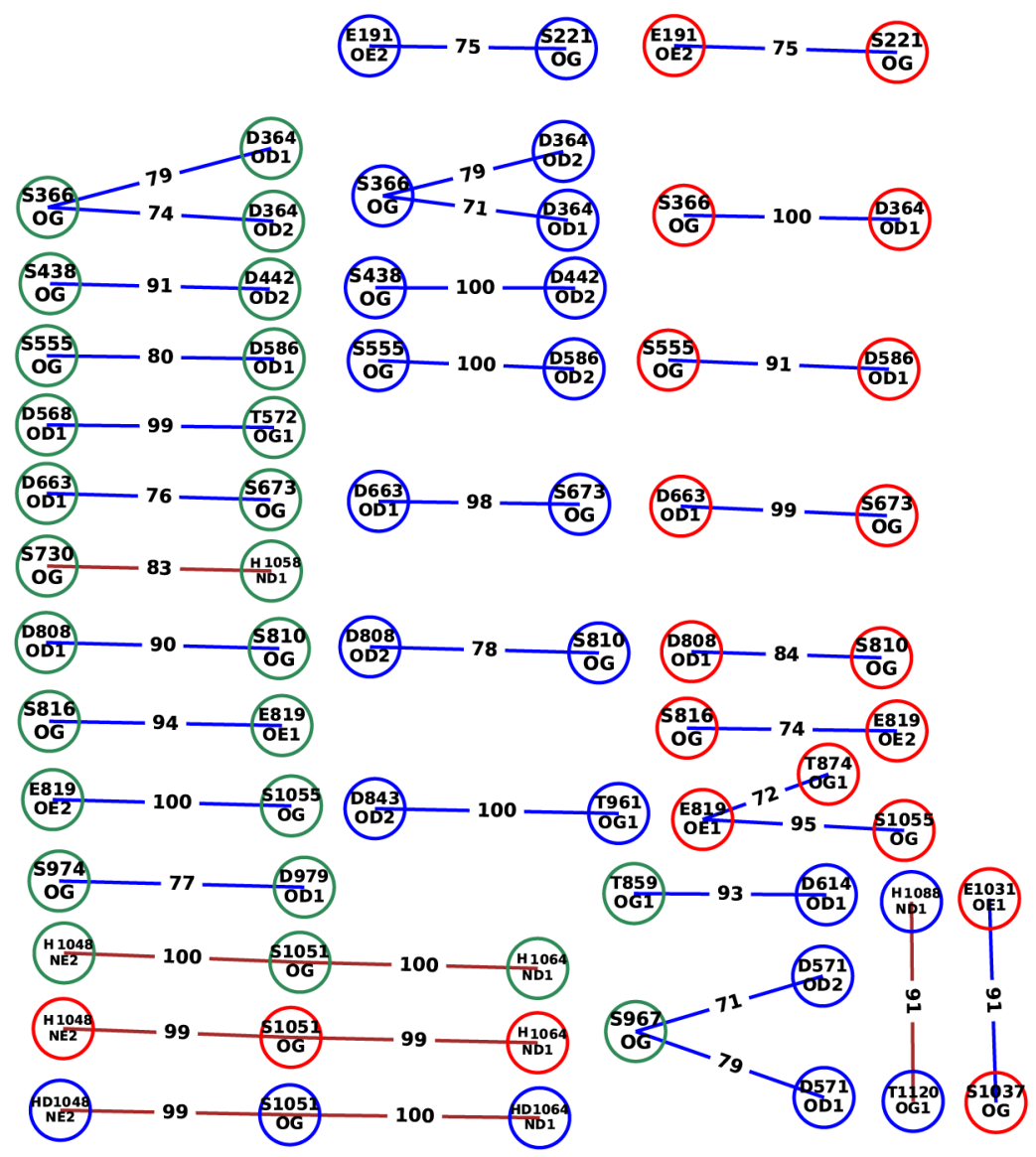

Figure S14. S/T - D/E hydroxyl - carboxylate motifs and $S / T-H$ hydroxyl - imidazole motifs identified with Bridge 2 and filtered at a minimum occupancy of $70 \%$. Nodes colored green, blue, and red indicate groups that belong to protomers $\mathrm{A}, \mathrm{B}$, and $\mathrm{C}$, respectively. For clarity of the graph, here and elsewhere in main text and Supporting Information Figures the filling colors of the nodes representing $\mathrm{H}$-bonding groups were removed manually. 


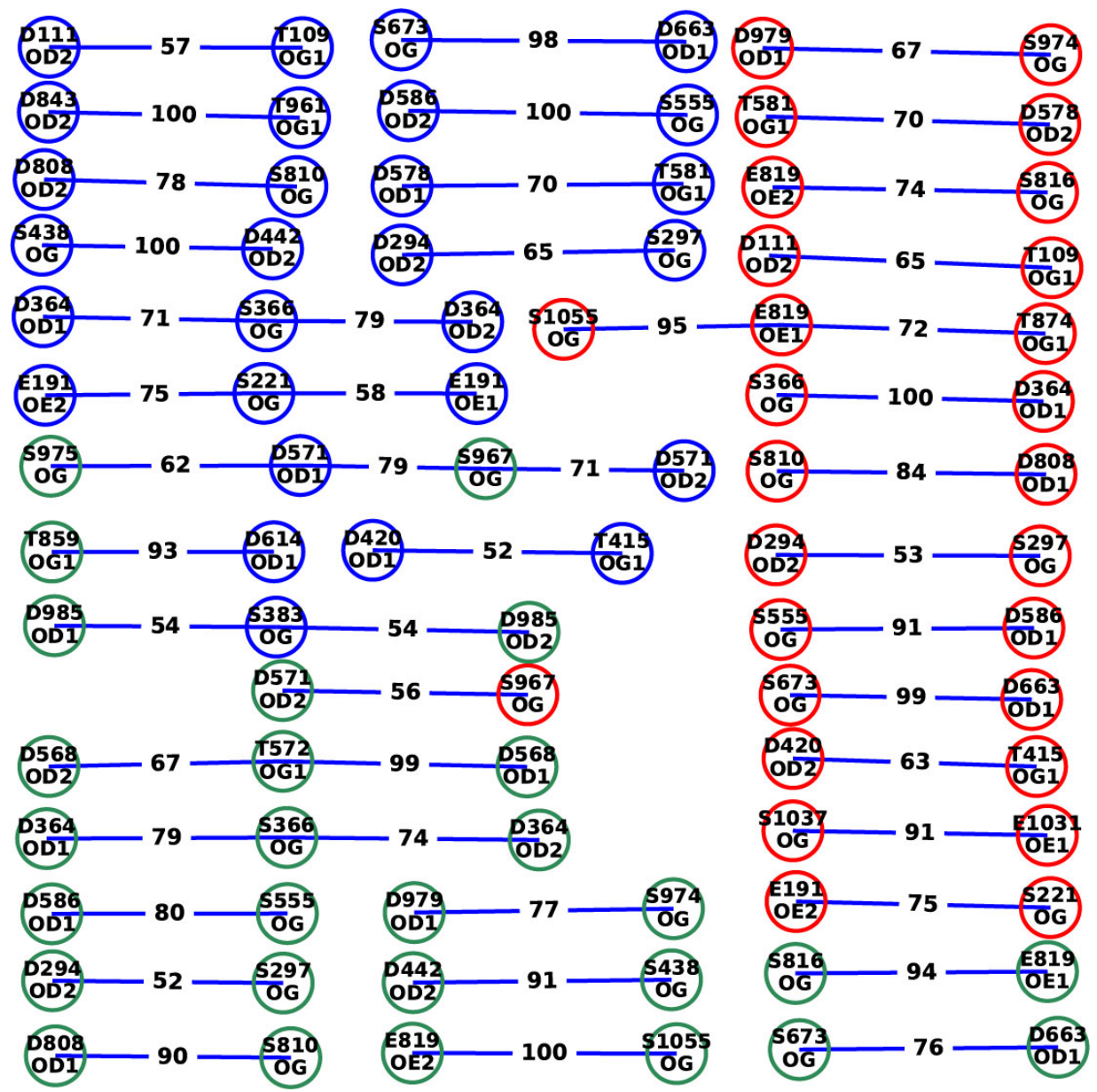

Figure S15. The graph for hydroxyl-carboxylate H-bond motifs identified at occupancy $\geq 50 \%$, with atom names for the nodes of the graph. Some of the $\mathrm{H}$-bonds presented here are also shown in the $70 \%$ occupancy graph from Figure S14. Note, for example, that the $\mathrm{H}$-bond between E191-OE2 and S221-OG of protomer A, present with $75 \%$ occupancy in the graph from Figure $\mathrm{S} 14$, is present in this graph together with the $\mathrm{H}$-bond between $\mathrm{E} 191$ OE1 and S221-OG, which has a lower occupancy of 58\%. 


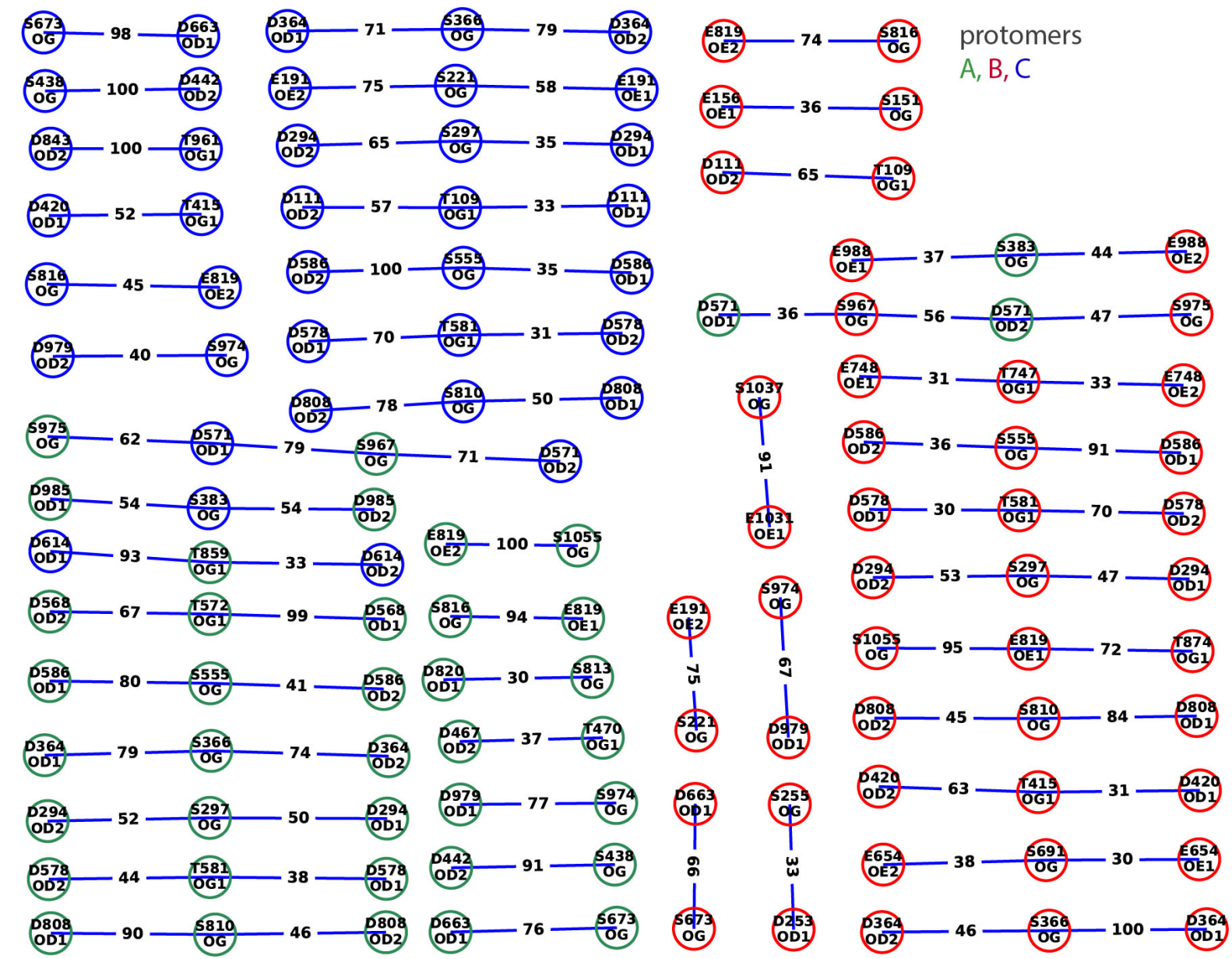

Figure S16. Illustration of the Ser/Thr-Asp/Glu hydroxyl-carboxyl H-bond motifs identified for protein S, with a minimum occupancy of at least $30 \%$. 


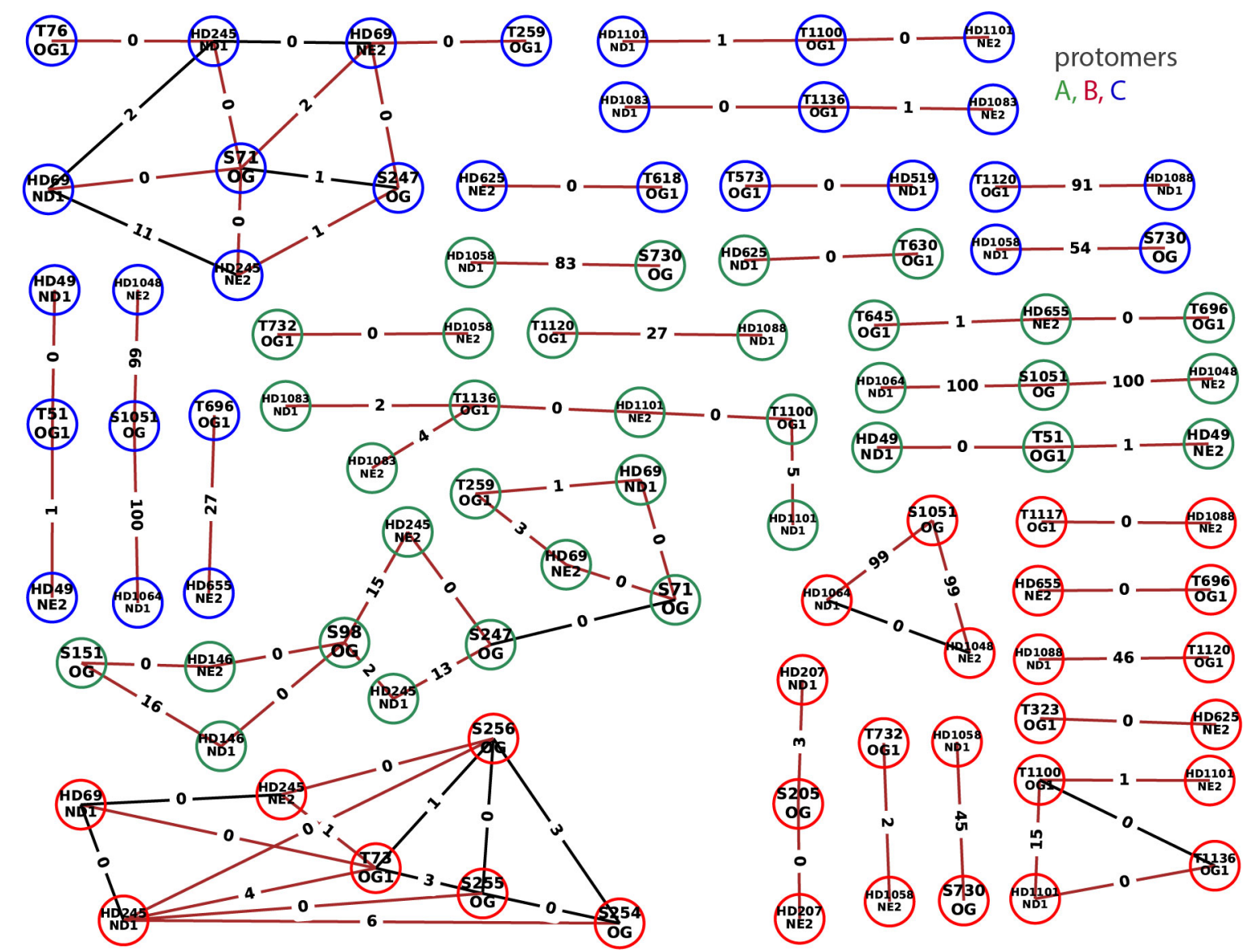

Figure S17. Graph of the Ser/Thr-His H-bonds without occupancy filter. Although most of these $\mathrm{H}$-bonds have low occupancies, several sites have high $\mathrm{H}$-bond occupancy, most notable the $\mathrm{H}$-bonds between $\mathrm{S} 1051, \mathrm{H} 1048$ and $\mathrm{H} 1064$, which are present in all three protomers with occupancies of $99-100 \%$. The graph for Ser/Thr-His H-bonds with occupancies of at least $50 \%$ is presented in Figure S18.

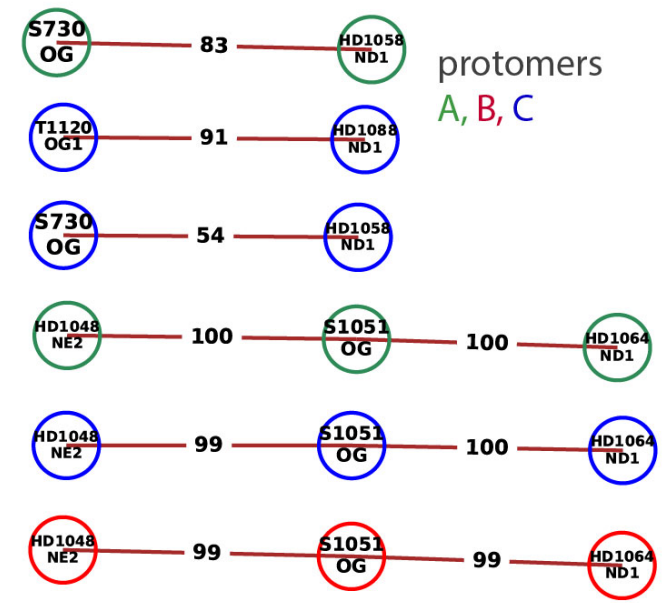

Figure S18. Graph of the Ser/Thr-His H-bonds with occupancies of at least $50 \%$. Note that, in all three protomers, there are highly stable H-bonds between S1051, H1048, and H1064. 


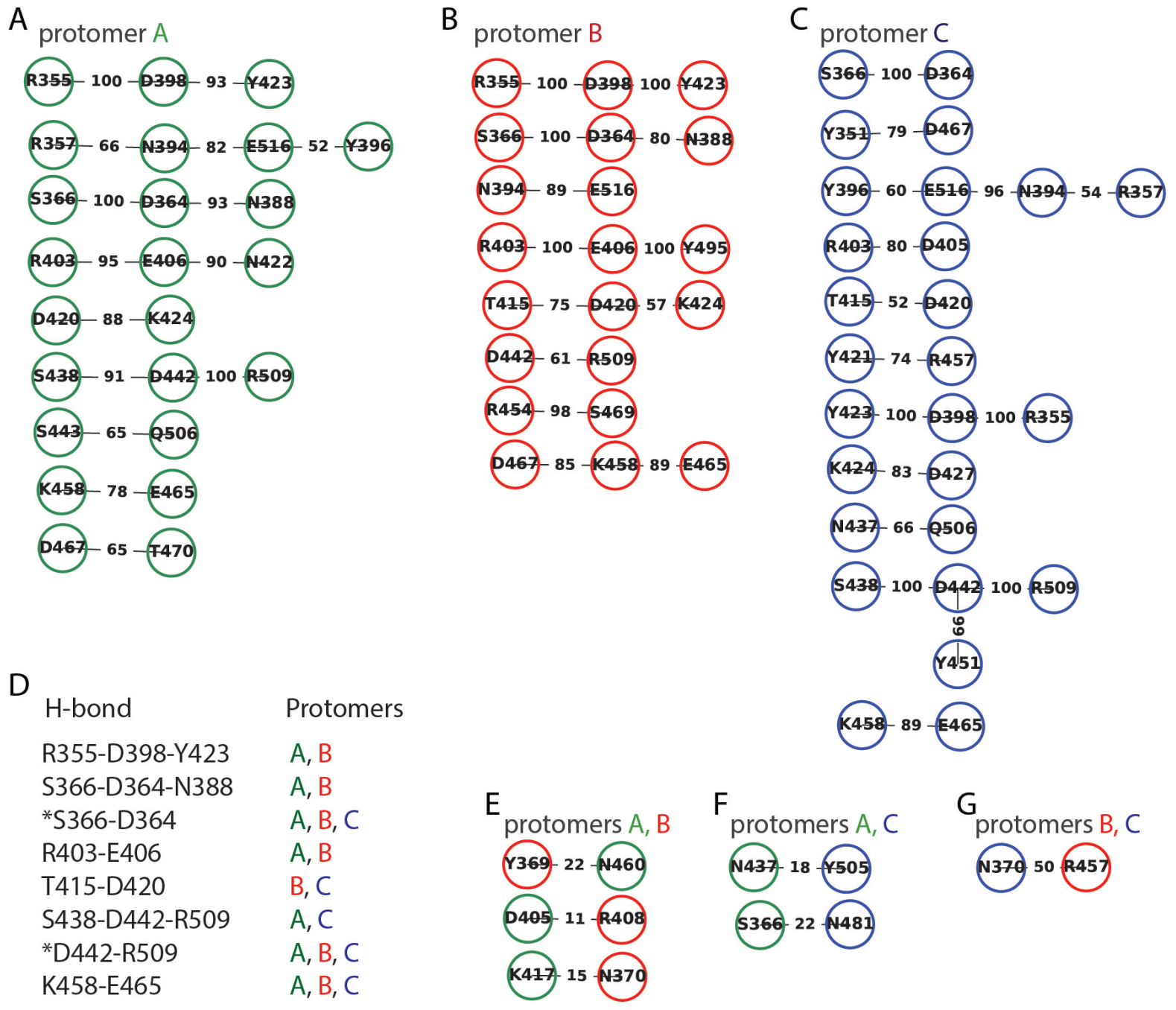

Figure S19. H-bonding within the RBDs of protein S, and between the RBDs. (A-C) H-bond maps computed for sidechains of each of the RBDs. H-bonds for sidechains of amino acid residues $331-527$ are shown at a minimum occupancy of $50 \%$. (D). Summary of H-bonds present in at least two of the RBDs. (E-G) H-bonds between RBDs of two different protomers, shown at a minimum occupancy of $10 \%$. H-bond graphs were computed with the Between Selections tool of the Bridge2 interface. 
A

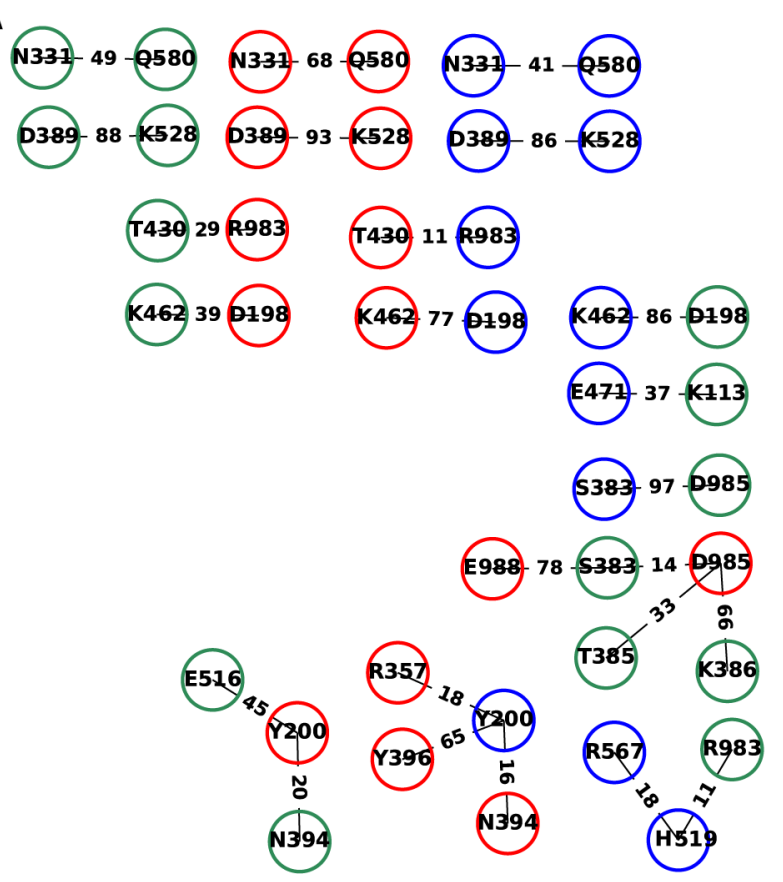

B

intra-protomer $\mathrm{H}$-bond

N331-Q580 A, B, C

D389-K528 A, B, C

inter-protomer $\mathrm{H}$-bond

T430-R983 A-B, B-C

K462-D198 A-B, B-C, A-C

Figure S20. $\mathrm{H}$-bonding between the three RBDs and the remaining of protein $\mathrm{S}$. (A) H-bond graph shown at a minimum occupancy of $10 \%$. (B) Summary of $\mathrm{H}$-bonds present in at least two of the protomers. 


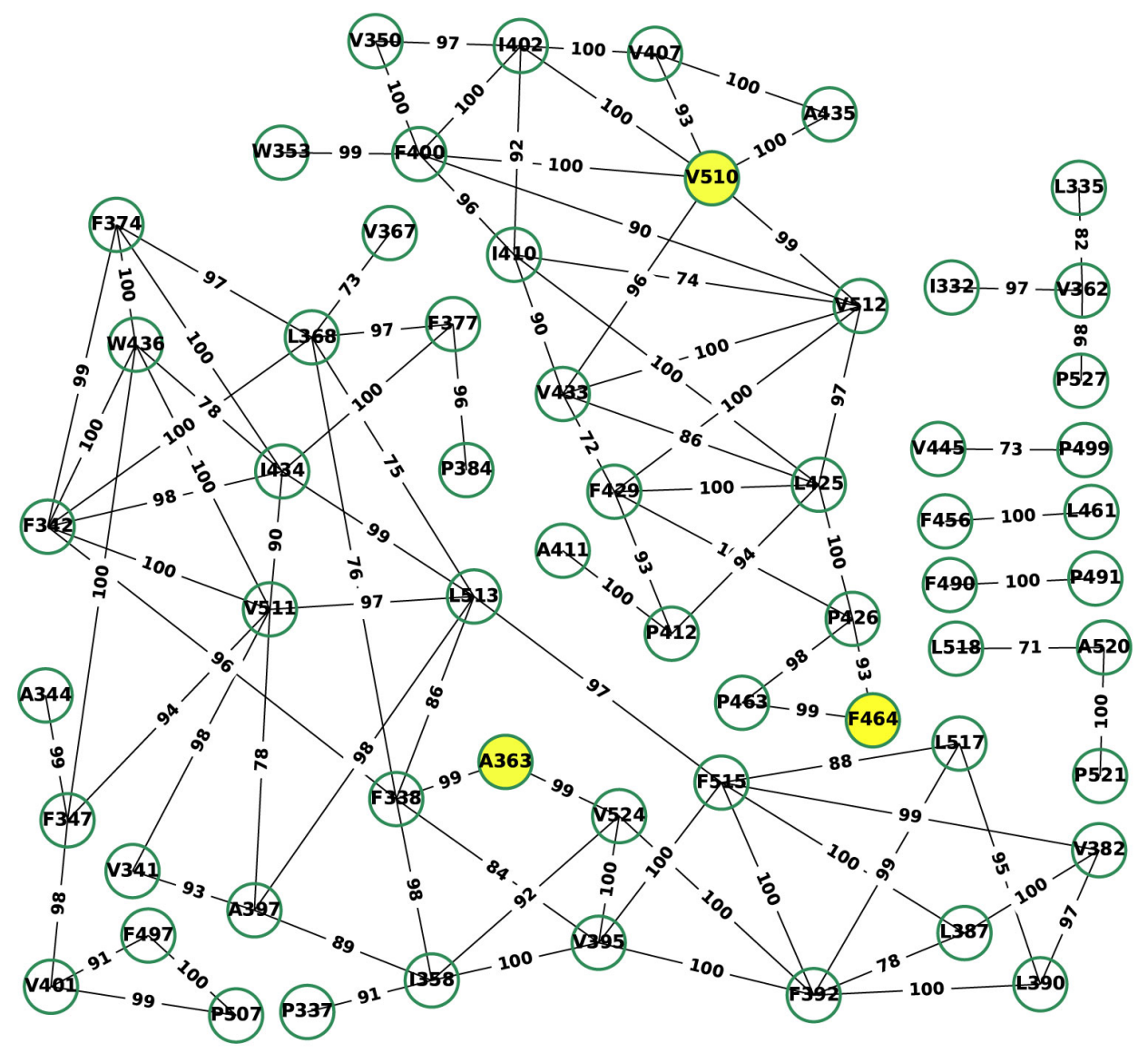

Figure S21. Hydrophobic contacts computed for the RBD of protomer A of protein S. Given the large number of hydrophobic contacts, for clarity, only hydrophobic contacts with occupancies $\geq 70 \%$ are shown. The three groups highlighted yellow are adjacent in the sequence to $\mathrm{H}$-bonding groups involved in persistent $\mathrm{H}$-bonds in each of the three protomers. 


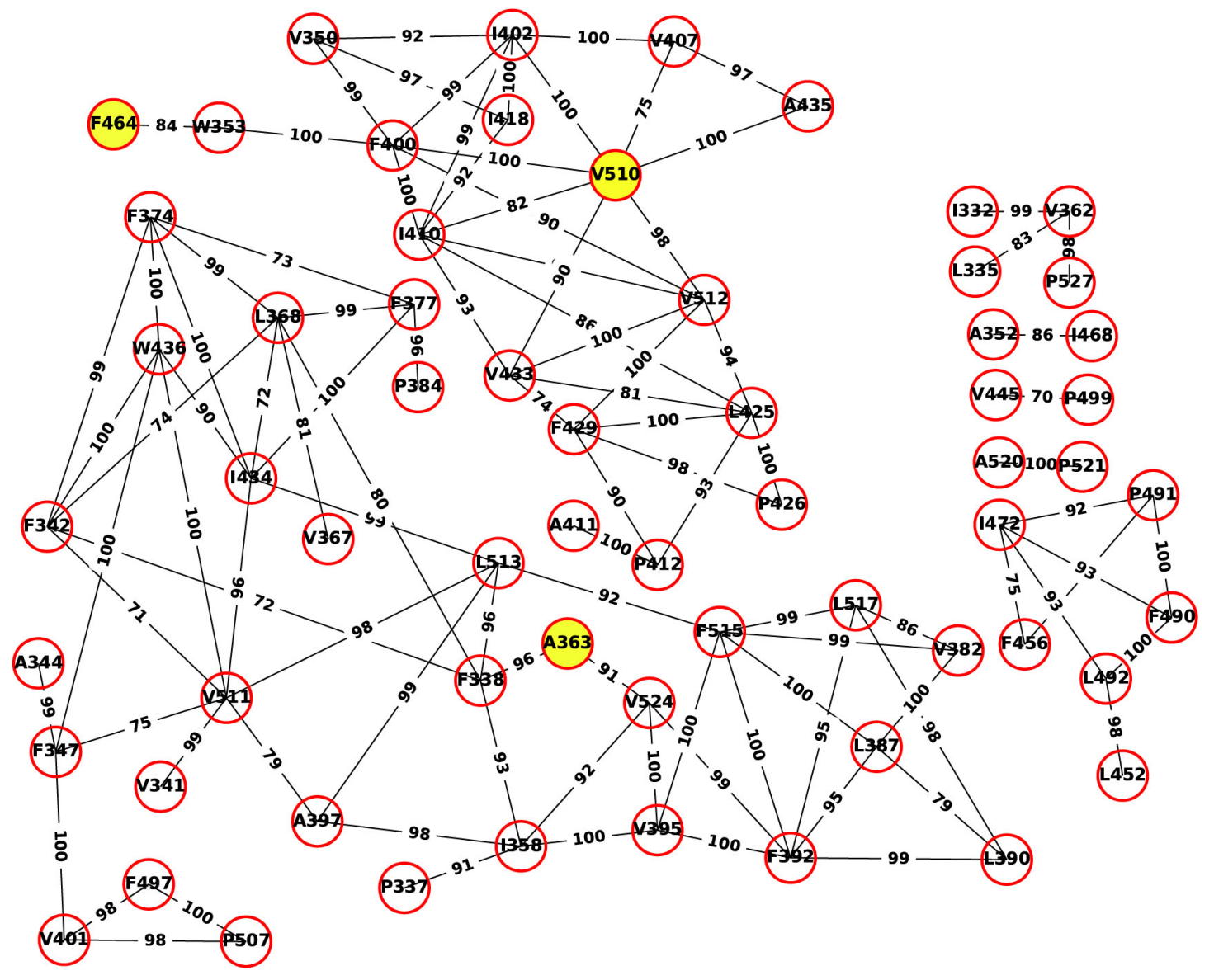

Figure S22. Hydrophobic contacts computed for the RBD of protomer B of protein S. The minimum occupancy of the hydrophobic contacts shown is $70 \%$. 


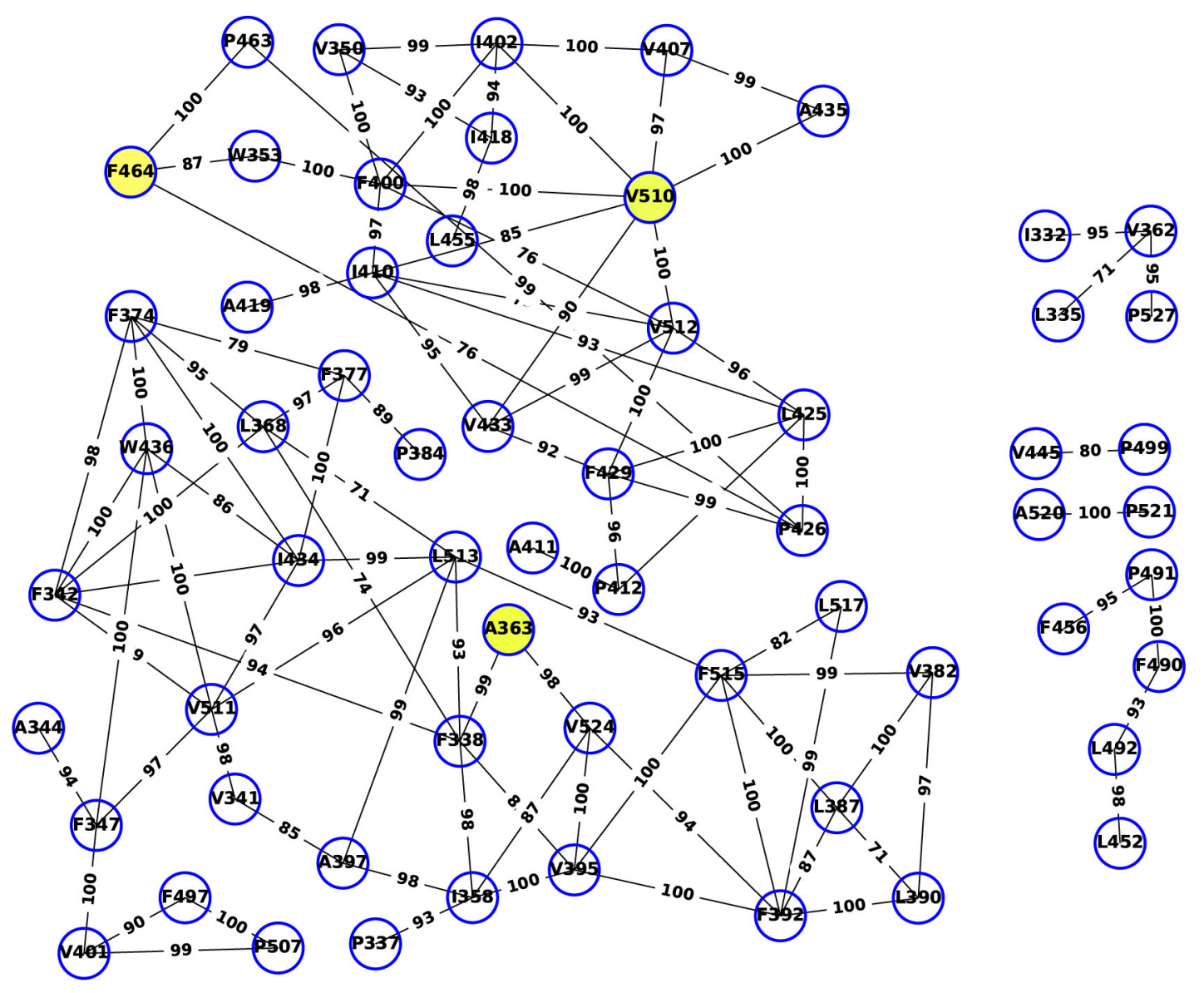

Figure S23. Hydrophobic contacts computed for the RBD of protomer $C$ of the spike protein. Contacts shown have a minimum occupancy of $70 \%$. 

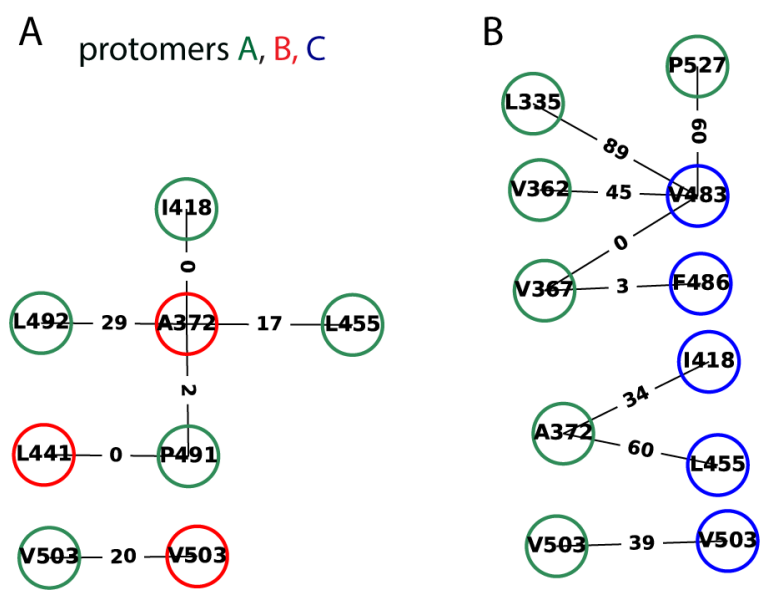

C

Figure S24. Hydrophobic contacts between RBDs of protein S. All contacts are shown, without an occupancy filter. (A-C) Hydrophobic contacts between protomers $A$ and $B$ (panel $A$ ), between protomers $A$ and $C$ (panel $B$ ), and between protomers $B$ and $C$ (panel $C$ ). Note that V503 of protomer A contacts V503 of protomers B and C with $20 \%$ or $39 \%$ occupancy, that is, these hydrophobic contacts are dynamic. As V503 is present as Val, lle or Leu in spike protein sequences, ${ }^{3}$ dynamic hydrophobic contacts at this site could be important for the protein. The contact between $\mathrm{A} 372$ and $\mathrm{L} 455$, connects protomers $\mathrm{A}$ and $\mathrm{B}$, and protomers B and C. A372 replaces a Thr group in SARS-CoV and bat protein S, and L455 substitutes for a Lys group in SARS-CoV protein $\mathrm{S}^{3}$ 
A

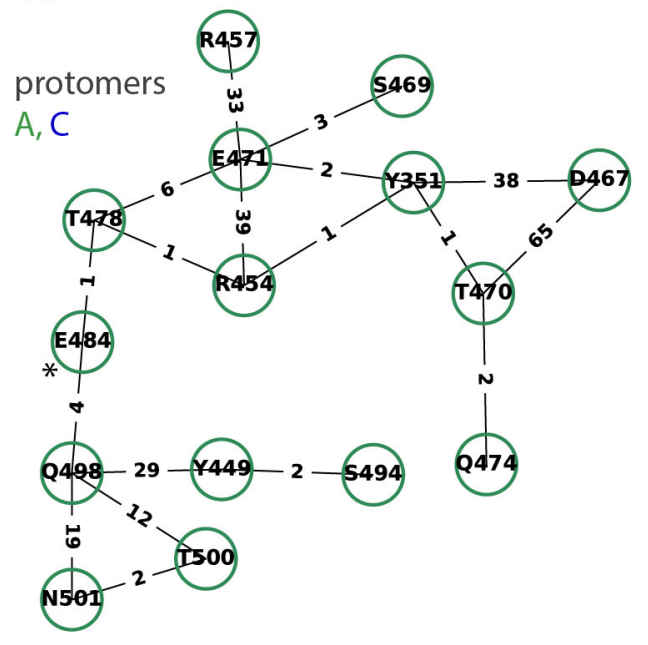

B

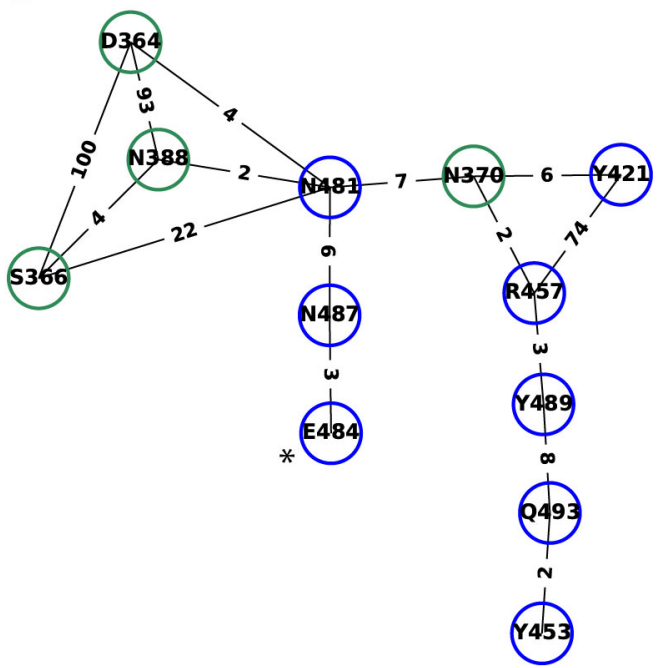

Figure S25. Local H-bond clusters identified for E484 of each protomer have only weak interactions between sidechains. The graph included $\mathrm{H}$-bonds with occupancies of at least $1 \%$. (A,B) H-bond clusters obtained with Connected Component Analyses using as a root node E484 of protomer A (panel A) or of protomer C (panel B).

A

protomers $\mathrm{A}, \mathrm{B}, \mathrm{C}$

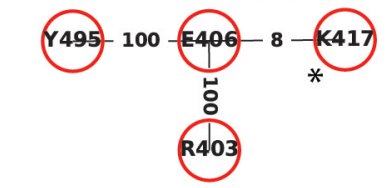

B

(-406- $42-417$

C<smiles>c1ccc([As]2CCCC2)cc1</smiles>

D

(4449- $32-8499-8$
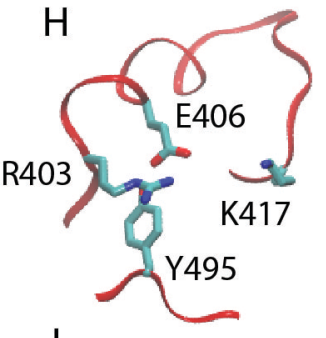

$\mathrm{L}$

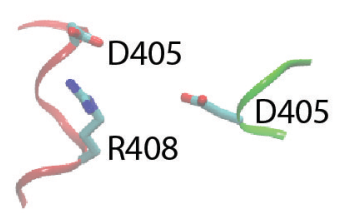

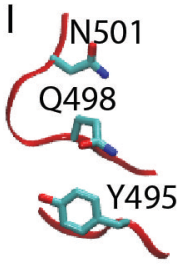

M

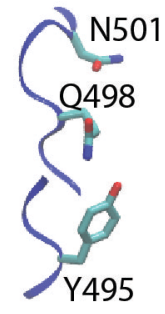

D405 $x^{\text {R408 }}$ K417

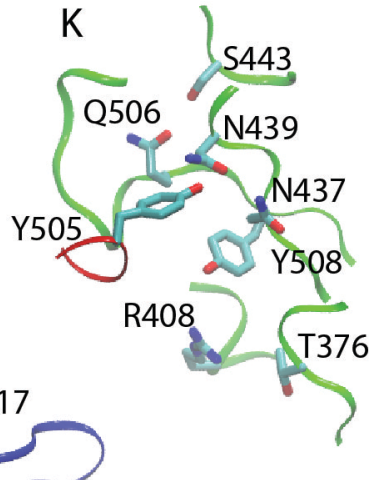

E 405

\section{$\mathrm{F}$}

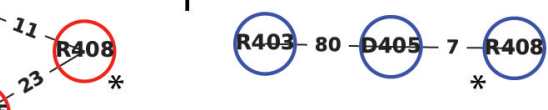

R403

G 2405

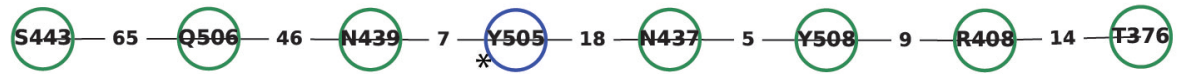

Figure S26. Selected local $\mathrm{H}$-bond clusters in the RBDs of protomers $B$ and $C$. $(A-H)$ Graphs of local H-bond clusters identified for K417 or protomers B (panel A) and C (panel $B$ ), N501 of protomers B (panel C) and C (panel D), R408 of protomers B (panel E) and C (panel F), and for $\mathrm{Y} 505$ of protomer $\mathrm{C}$. (H-M) Molecular graphics of local H-bond clusters identified in panels A-G. Note that clusters identified for R408 and K417 of protomer B (panels $\mathrm{B}, \mathrm{F}$ ) are illustrated in panel $\mathrm{M}$. 


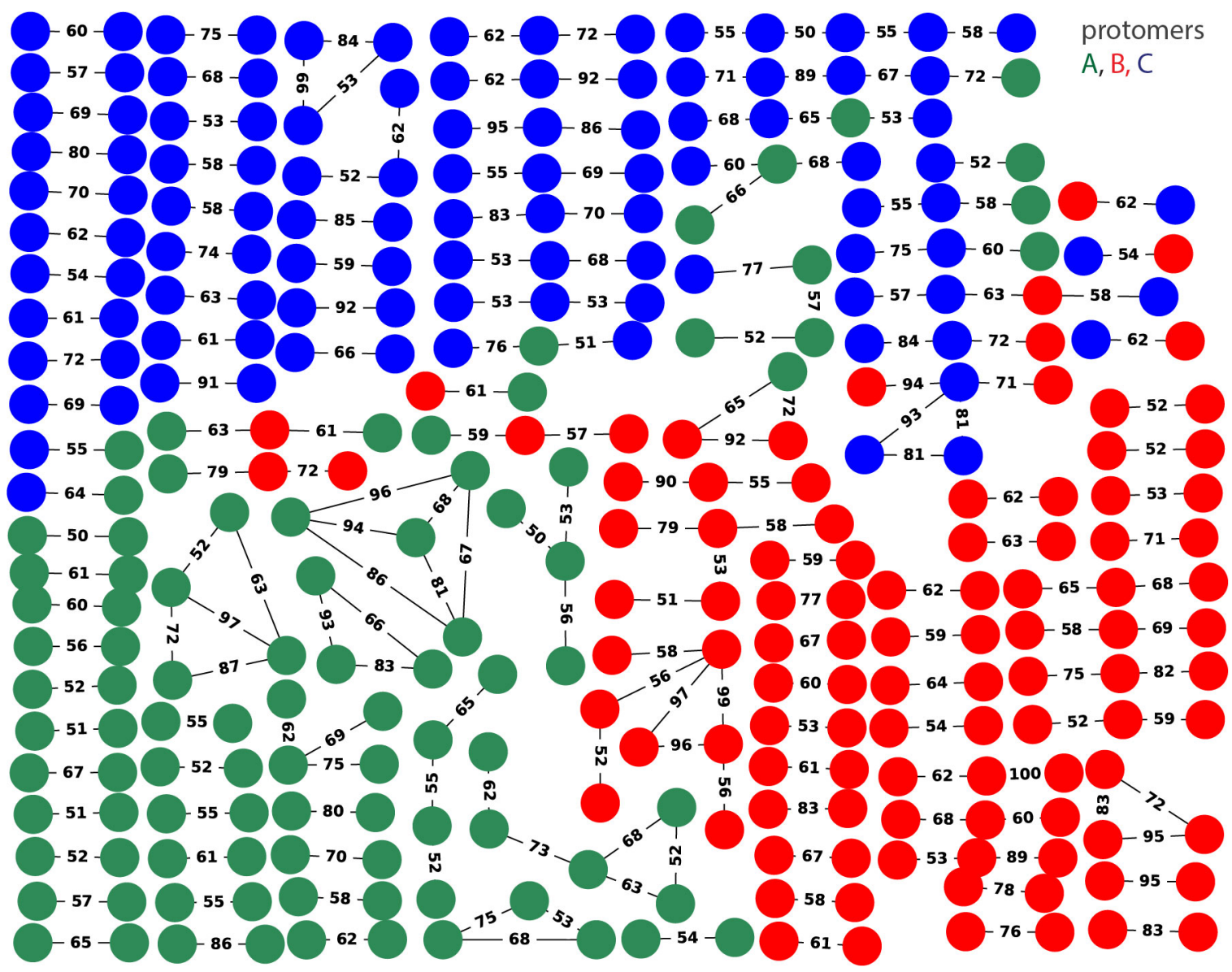

Figure S27. Water-mediated bridges between sidechains of protein S, computed with one water in the bridge and filtered at a minimum of $50 \%$ occupancy. For clarity, amino acid residue numbers are not shown. Numbers along graph edges indicate the occupancy of the bridge. Detailed views of selected water-mediated interactions contained in this graph are shown in Figure S28. 
protomers $\mathrm{A}, \mathrm{B}, \mathrm{C}$

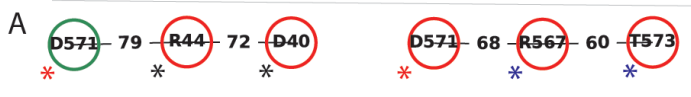

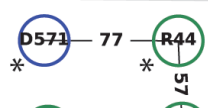
* 1492 - 540
* 53 * 53 (040
*

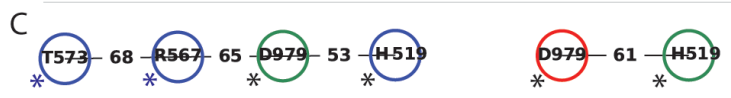

D

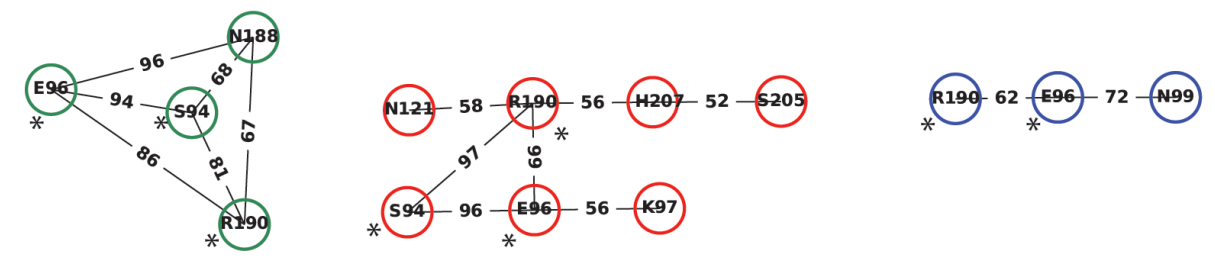

E

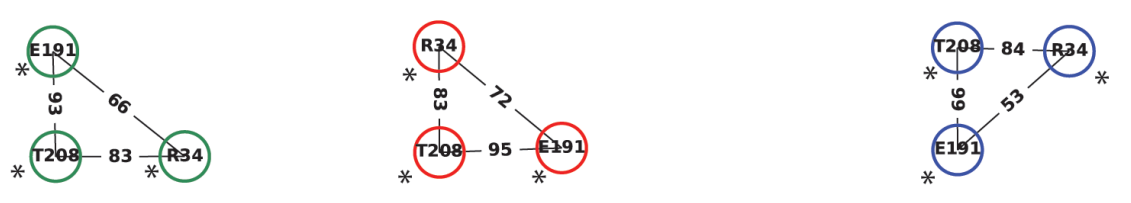

F
(129) 50 - 2215
*266- 52 (995 59
*213) 68 *266

G

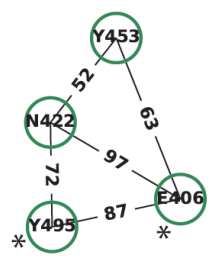

(4995) 58 - 500

$\mathrm{H}$

(335)- 62 - 5514

I

(466) 55 -4454 69

(558) $52-652-508$

J

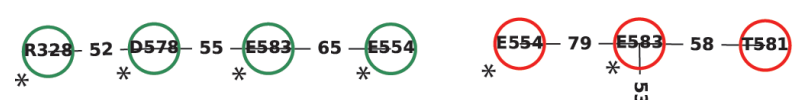

$$
\begin{aligned}
& \text { * } 3328-51 \text { * }
\end{aligned}
$$

K<smiles>c1ccc([Se]c2cccc(-[13c]3[13cH][13cH][13cH][13cH][13cH]3)c2)cc1</smiles> 


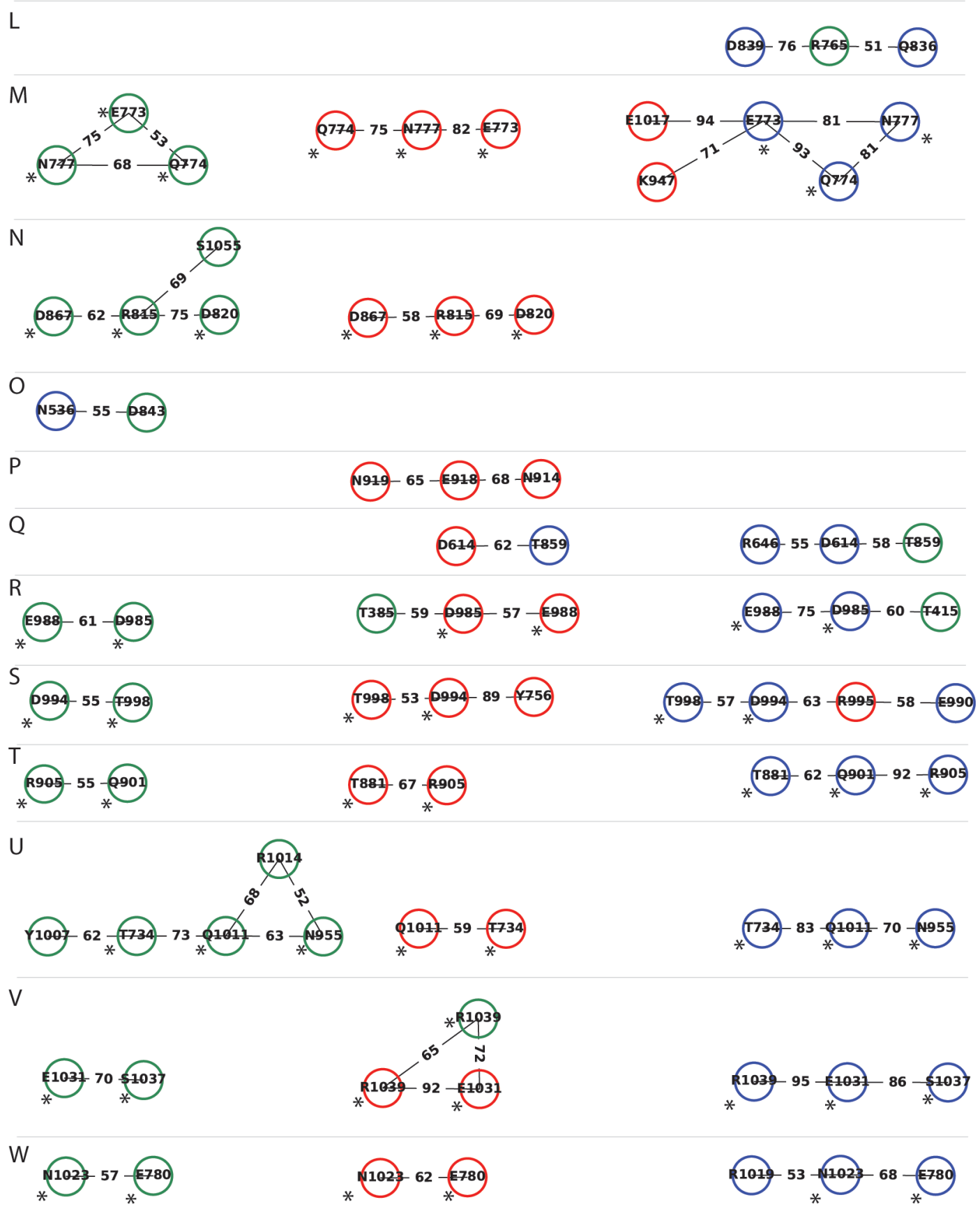

Figure S28 Close view of local H-bond clusters extracted from the $\mathrm{H}$-bond graph of onewater bridges filtered at $50 \%$ in Figure S27. The star signs indicate protein groups that participate in $\mathrm{H}$-bond clusters in at least two protomers. (A) $\mathrm{H}$-bond graphs for selected local interactions of D571 of each protomer. (B) Local H-bonding for D568 (panel B), D979 (panel C), E96 (panel C), E96 (panel D), E191 (panel E), D215 and Y266 (panel F), E406 (panel G), D398 (panel H), E465 and D586 in protomer C (panel I), D578 (panel J), D737 (panel K), D839 (panel L), E773 (panel M), D820 (panel N), D843 (panel O), E918 (panel P), D614 (panel Q), D985 and E988 (panel R), D994 (panel S), R905 (panel T), Q1011 (panel U), E1031 (panel V), and E780 (panel W). Note that H-bond clusters presented in panels A-R and U-V include one or more than one carboxylate groups. 


\section{References}

1. Gowers, R. J.; Linke, M.; Barnoud, J.; Reddy, T. J. E.; Melo, M. N.; Seyler, S. L.; Dotson, D. L.; Domanski, J.; Buchoux, S.; Kenney, I. M.; Beckstein, O., MDAnalysis: A Python Package for the Rapid Analysis of Molecular Dynamics Simulations. S. Benthall and S. Rostrup, Editors, Proceedings of the 15th Phyton in Science Conference, Austin, TX, 2016 SciPy 2016, 102-109.

2. Humphrey, W.; Dalke, W.; Schulten, K., VMD: Visual Molecular Dynamics. J. Mol. Graph. 1996, 14, 33-38.

3. Jaimes, J. A.; André, N. M.; Chappie, J. S.; Millet, J. K.; Whittaker, G. R., Phylogenetic Analysis and Structural Modeling of SARS-CoV-2 Spike Protein Reveals an Evolutionary Distinct and Proteolytically Sensitive Activation Loop. J. Mol. Biol. 2020, 432, 3309-3325. 